\title{
SAFT- $\gamma$ Force Field for the Simulation of Molecular Fluids. 1. A Single-Site Coarse Grained Model of Carbon Dioxide
}

\author{
Carlos Avendaño, Thomas Lafitte, Amparo Galindo, Claire S. Adjiman, George Jackson, and Erich A. Müller*
}

Department of Chemical Engineering, Centre for Process Systems Engineering, Imperial College London, South Kensington Campus, London SW7 2AZ, U.K.

ABSTRACT: An application of the "top-down” concept for the development of accurate coarse-grained intermolecular potentials of complex fluids is presented. With the more common "bottom-up" procedure, coarse-grained models are constructed from a suitable simplification of a detailed atomistic representation, and small adjustments to the intermolecular parameters are made by comparison with limited experimental data where necessary. In contrast, in the top-down approach, a molecular-based equation of state is

\section{SAFT - $\gamma$ Mie force field}

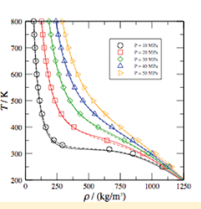

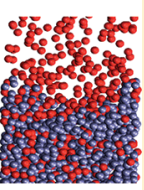

coarse grained simulation used to obtain an effective coarse-grained intermolecular potential that reproduces the macroscopic experimental thermophysical properties over a wide range of conditions. These coarse-grained intermolecular potentials can then be used in conventional molecular simulation to obtain properties (such as structure or dynamics) that are not directly accessible from the equation of state or at extreme conditions where the theory is expected to fail. To demonstrate our procedure, a coarse-grained model for carbon dioxide $\left(\mathrm{CO}_{2}\right)$ is obtained from a recent implementation of the statistical associating fluid theory of variable range (SAFT-VR) employing a Mie (generalized Lennard-Jones) potential; the parameters of this single-site Mie model of $\mathrm{CO}_{2}$ are estimated by optimizing the equation of state's description of the experimental vapor-pressure and saturated liquid density data. This approach is only viable because of the excellent agreement of the SAFT-VR Mie EoS with simulation data. Our single-site SAFT- $\gamma$ coarsegrained model for $\mathrm{CO}_{2}$ is used in Monte Carlo molecular simulation to assess the adequacy of the description of the fluid-phase behavior and properties that were not used to develop the potential model such as the enthalpy of vaporization, interfacial tension, density profiles, supercritical densities, and second-derivative thermodynamic properties (thermal expansivity, isothermal compressibility, heat capacity, Joule-Thompson coefficient, and speed of sound). The accuracy of the description with the single-site SAFT- $\gamma$ model of $\mathrm{CO}_{2}$ is found to be of similar quality to that of more sophisticated intermolecular potentials such as a six-site (three LJ centers and three charged sites) all-atom model. The SAFT- $\gamma$ top-down approach to coarse-graining resolves a key challenge with coarse-graining techniques: the provision of a direct robust link between the microscopic and macroscopic scales.

\section{INTRODUCTION}

Detailed all-atom or united-atom models (e.g., the OPLS ${ }^{1}$ or TraPPE-type ${ }^{2}$ force fields) are now in ubiquitous use in computer simulation of molecular fluids and are often capable of describing molecular systems with a precision that supplements experiments. More than $1 \%$ of all the recent articles published in the open scientific and engineering literature involve molecular simulation at this level. ${ }^{3}$ Exponential, Moore-law type,${ }^{4}$ increases in computational hardware have extended the limits of possibilities in simulations of large systems; one of the recent records is the molecular dynamics simulation of $3.2 \times 10^{11}$ Lennard-Jones (LJ) atoms of a copper crystal cube with $1.56 \mu \mathrm{m}$ edge lengths, ${ }^{5}$ followed only two years later by the simulation of a system that was an order of magnitude larger. ${ }^{6}$ In spite of these impressive metrics and forecasts for the future, molecular simulations spanning even larger length and time scales are routinely required, and coarsegraining (CG) methods must be developed to bridge the gap between the atomistic modeling of matter and the commonplace continuum description of fluids and solids. ${ }^{7}$

Highlights in the modeling of soft matter using CG techniques have been collected in three recent volumes: a book edited by Voth, ${ }^{7}$ and themed issues of Physical Chemistry Chemical Physics, ${ }^{8}$ and Faraday Discussions of the Royal Society of Chemistry. ${ }^{9}$
Excellent reviews on the topic have also recently appeared, such as those by Klein and Shinoda ${ }^{10}$ and McCullagh et al., ${ }^{11}$ to name but a salient few. There are two key challenges faced by any coarse-graining scheme: robustness and transferability. First of all, a formal connection must be established between the coarsegrained model and the underlying (full resolution) model that one is attempting to describe. The resulting description must then be transferable to other similar systems not used in the initial parametrization of the CG model. The resulting model must be robust, with a clear connection between the detailed molecular and macroscopic information, and it must be reliable, allowing for predictions at conditions removed from those where the model was developed.

The techniques that have been used to address these challenges with a certain degree of success have by and large followed a "bottom-up" method. This approach to coarse-graining consists of matching the properties of either a classical atomistic model or a quantum model with those of a "superatom". At this level of description, the CG methodology generally consists of
Received: May 26, 2011
Revised: $\quad$ August 2, 2011
Published: August 04, 2011 
removing some of the degrees of freedom in the system to simplify its description, while at the same time attempting to maintain the thermodynamic description. The theoretical framework for this procedure has a firm and well established foundation. ${ }^{12}$ From a statistical mechanical point of view, the full thermodynamic description of a system can be obtained once the Helmholtz free energy $A$ of a system is determined. There is a direct relationship between $A$ and the corresponding configurational integral:

$$
\begin{aligned}
& \exp (-\beta A)=C \int_{V} \exp [-\beta U(r)] d r \\
& \approx C^{\prime} \int_{V} \exp \left[-\beta U_{C G}\left(r_{C G}\right)\right] d r_{C G}
\end{aligned}
$$

where $U(\mathbf{r})$ refers to the total intermolecular potential that is a function of the vector of all relevant configurational variables $\mathbf{r}, \beta$ $=1 / k_{\mathrm{B}} T, k_{\mathrm{B}}$ is the Boltzmann constant, $T$ is the temperature, and $C$ and $C^{\prime}$ are specific constants that incorporate the kinectic contributions. The aim and "holy grail" of CG techniques is to be able to reduce the full phase space of $\mathbf{r}$ to a small subspace of these, $\mathbf{r}_{\mathrm{CG}}$, in such a way that the solution of the configurational integral of the new CG model (and its new intermolecular potential energy, $U_{\mathrm{CG}}\left(\mathbf{r}_{\mathrm{CG}}\right)$ ) represents in the optimal way the original free energy of the system. This statement is a sufficient condition to achieve consistency in configurational space. ${ }^{12}$ Despite the beguiling simplicity of the relationship between the full and CG models, the implementation of the procedure is far from trivial. The exact solution of the configurational integral is impossible apart from the most trivial cases, so the methodology that is used consists of performing a limited molecular simulation study of the full system (eq 1a). The resulting data is then taken as that of the "real" or full-resolution model, and its properties are used to construct the CG model as described by eq $1 \mathrm{~b}$.

A wide range of properties are used as the "target" in this context including the matching of effective forces between molecules, ${ }^{13,14}$ molecular structure (using iterative Boltzmann inversion), ${ }^{15,16}$ interfacial tensions, ${ }^{17}$ partitioning free energies between polar and apolar phases (partition coefficients), ${ }^{18}$ and/ or critical points, ${ }^{19-25}$ or maximizing the overlap between the target and CG distributions (maximizing the so-called relative entropy). ${ }^{26}$ In most of these cases, the robustness of the methods is guaranteed by following well founded statistical mechanical recipes. However, a critical inspection of eq 1 leads one to the conclusion that the transferability to other thermodynamic states and systems will be limited when one adheres to the correct procedure. By eliminating degrees of freedom, we are not capable of uniquely describing the full free energy landscape. The problem plaguing many of the aforementioned methods is that the resulting intermolecular potentials are state and system specific. The issue of transferability is still elusive and has been addressed by several groups in terms of a group contribution approach, where molecules are represented as an assembly of functional building blocks interacting in the same way for different systems. Methodologies using this approach are the MARTINI force field ${ }^{18}$ (biomolecular systems), the CG force fields of Klein and co-workers ${ }^{15,17,27,28}$ (alkanes, biomolecular systems, amino acids, phenyl-based molecules), the CG force field of Chiu et al. ${ }^{29}$ (water and alkanes), and the CG version of the TraPPE force field (TraPPE-CG) developed by Maerzke and Siepmann (alkanes). ${ }^{30}$ Different functional forms are used for the nonbonded interactions in these CG force fields. In the MARTINI force field the nonbonded interactions are parametrized with a simple LJ potential. However, for the other methodologies, a more flexible functional form, which allows a greater control of the fine balance between the attractive and repulsive contributions, is used by means of the Morse or Mie potentials.

In this contribution we propose a direct route to obtaining the required CG potential from macroscopic thermodynamic data. Our "top-down" approach relies on having access to an accurate molecular-based equation of state (EoS) that describes the Helmholtz free energy in closed algebraic form and is formulated explicitly in terms of a predefined intermolecular potential. An equation of state of this type can be used to explore a wide parameter space to estimate the set of intermolecular potential parameters that provides an optimal description of the macroscopic experimental data. This parameter set does not represent a unique mapping to a single state point (temperature or density), but rather an overarching average over the entire regression space. When the EoS is expressed in terms of the free energy of the system for a well-defined intermolecular potential, it can be used to develop a "top-down averaged" CG intermolecular potential.

We follow this vein here and present a proof-of-concept of our top-down approach for carbon dioxide $\left(\mathrm{CO}_{2}\right)$, employing the accurate and versatile family of EoSs based on the statistical associating fluid theory for potentials of variable range (SAFT$\mathrm{VR})^{31,32}$ as developed for the Mie (generalized LJ) force field. ${ }^{33,34}$ The SAFT-VR Mie EoS is used to develop a SAFT- $\gamma$ $\mathrm{CG}$ force field for $\mathrm{CO}_{2}$ by estimating the parameters from fluidphase equilibrium data for the vapor-pressure and saturated liquid density. We adopt the more general SAFT- $\gamma$ terminology to describe our methodology because it refers to the formulation of SAFT-VR as a generic group contribution approach, ${ }^{35-37}$ which allows for a united atom or CG representation on an equal footing.

\section{SAFT- $\gamma$ FORCE FIELD: MIE POTENTIAL AND SAFT-VR MIE EOS}

The coarse-graining strategy used in our work is based on the assumption that a wide range of real substances can be modeled effectively as chain molecules formed from fused Mie segments. The use of this type of generalized Lennard-Jonesium potential as the elementary building block for homonuclear chains of spherical segments has proven to be very fruitful in modeling simultaneously the fluid behavior and second-derivative thermodynamic properties of a wide variety of systems. ${ }^{33,34}$ The $\left(\lambda_{\mathrm{r}}, \lambda_{\mathrm{a}}\right)$ Mie potential acting between two spherical segments can be expressed as ${ }^{38,39}$

$$
u^{\mathrm{Mie}}(r)=O \varepsilon\left[\left(\frac{\sigma}{r}\right)^{\lambda_{\mathrm{r}}}-\left(\frac{\sigma}{r}\right)^{\lambda_{\mathrm{a}}}\right]
$$

where $r$ is the intersegment distance, $\varepsilon$ is the potential depth, $\sigma$ is the position at which the potential is zero (segment diameter), and $\lambda_{\mathrm{r}}$ and $\lambda_{\mathrm{a}}$ are the repulsive and attractive exponents which characterize the pair energy. The constant $O$ in eq 2 is defined as

$$
\mathcal{G}=\frac{\lambda_{\mathrm{r}}}{\lambda_{\mathrm{r}}-\lambda_{\mathrm{a}}}\left(\frac{\lambda_{\mathrm{r}}}{\lambda_{\mathrm{a}}}\right)^{\lambda_{\mathrm{a}} /\left(\lambda_{\mathrm{r}}-\lambda_{\mathrm{a}}\right)}
$$

which ensures that the minimum of the potential corresponds to $-\varepsilon$. Once the intermolecular potential is defined, the challenge is to derive a closed analytical form for the Helmholtz free energy 
Table 1. Intermolecular Model Parameters for Single-Site LJ Model for $\mathrm{CO}_{2}$, Regressed from Different Properties and Data Sets

\begin{tabular}{llll}
\multicolumn{1}{c}{ property } & $\sigma / \AA$ & $\left(\varepsilon / k_{\mathrm{B}}\right) / \mathrm{K}$ & \multicolumn{1}{c}{ source $^{1}$} \\
viscosity & 3.881 & 216.06 & Ruckenstein and Liu $^{60}$ \\
viscosity & 3.941 & 195.20 & Reid et al. $^{59}$ \\
viscosity & 4.018 & 194.70 & Reed and Gubbins $^{58}$ \\
second virial coefficient & 4.416 & 192.25 & Liu et al. $^{61}$ \\
combined viscosity & 3.832 & 230.56 & Liu et al. $^{61}$ \\
\multicolumn{1}{c}{ and second virial coefficient } & & & \\
self-diffusion coefficient & 3.26192 & 500.71 & Liu et al. $^{61}$ \\
self-diffusion coefficient & 3.660 & 235.56 & Yu and Gao $^{62}$ \\
self-diffusion coefficient & 3.6283 & 195.20 & Dariva et al. $^{63}$ \\
critical properties & 3.912 & 225.30 & Iwai et al. $^{64}$ \\
critical properties & 3.644 & 231.70 & Zhu et al. $^{65}$ \\
critical properties & 3.658 & 232.20 & Albo and Müller $^{20}$ \\
volumetric properties & 3.720 & 236.10 & Iwai et al. $^{66}$ \\
\hline
\end{tabular}

of the system without loss of accuracy. The SAFT-VR equation of state is well suited for this purpose. For more details of the underlying SAFT approach the reader is referred to the original papers $^{40,41}$ and to various reviews of the method. ${ }^{42-45}$ The direct link between SAFT and the underlying potential has been explored before. ${ }^{46}$ The original versions of SAFT were developed to describe molecules with potentials of fixed form such as the LJ. The SAFT-VR formulation ${ }^{31,32}$ allows for a generic description of the effect of the range of the interaction and has been implemented for the square-well, ${ }^{31}$ Sutherland, ${ }^{31}$ LennardJones, ${ }^{47}$ and Yukawa ${ }^{48}$ potentials. More recently Lafitte et al. ${ }^{33}$ developed a version of the theory for chains of segments interacting through the Mie potential (SAFT-VR Mie) in closed analytical form. The general SAFT form of the Helmholtz free energy of a nonassociating chain fluid can be written as

$$
a=a^{\mathrm{IDEAL}}+a^{\mathrm{MONO}}+a^{\mathrm{CHAIN}}
$$

where $a=A / N k T$ is the dimensionless Helmholtz free energy, $a^{\text {IDEAL }}$ is the ideal free energy, $a^{\text {MONO }}$ is the residual free energy due to the monomer segments, and $a^{\text {CHAIN }}$ is the contribution due to the formation of chains of monomers. The reader is referred to our recent paper $^{34}$ for the latest developments with the SAFT-VR Mie EoS, and to the Appendix for a summary of the explicit relations used in our current work.

The Mie potential has long been recognized to allow for an improved description of different properties in both molecularbased theories and atomistic and CG simulations, when the Lennard-Jones potential is found to fail. It is well-known that force fields for the $n$-alkanes based on the LJ potential cannot be used to reproduce simultaneously the saturated liquid densities and vapor pressures of the fluid with good accuracy (see for example ref 49). In this respect the Mie potential is more flexible for the modeling of thermodynamic and transport properties as appropriate values of the repulsive (softness/hardness) and attractive exponents can be chosen to provide the best overall representation of the macroscopic properties. ${ }^{50,51}$ Early on Gibbons and Klein ${ }^{52}$ proposed a two-center model to study the thermodynamic properties of $\mathrm{CO}_{2}$ in the solid state using the Mie potential. In their work they found that by varying the repulsive exponent a very good description of the molar volume at absolute zero, the heat of sublimation, and the bulk elastic modulus can be achieved. More recently, Potoff and BernardBrunel $^{49}$ developed a new force-field for phase equilibrium calculations of the $n$-alkanes and $n$-perfluoroalkanes based on Mie united-atom segments. Using this force-field, the simultaneous representation of saturated liquid densities and vapor pressures for pure components and mixtures is possible. Related studies on the dynamical properties have been reported by Gordon, ${ }^{51}$ where the softness of the intermolecular potential was varied to obtain a better description of the viscosity of the $n$-alkanes.

The Mie potential has also been used in the development of CG force fields. For example, Nielsen et al. ${ }^{17}$ employed a CG model for $n$-alkanes parametrized to reproduce surface tension data using a $(9,6)$ Mie potential. He et al. ${ }^{53}$ followed a similar approach to obtain Mie potential parameters for the calculation of the surface tension in CG models of water. Klein and coworkers ${ }^{15,54,55}$ has now developed force fields based on the Mie potential to describe the nonbonded interaction for use in CG simulations to study the self-assembly of phospholipids ${ }^{15,54}$ and diblock copolymers. ${ }^{55}$ An equivalent methodology has been used to model ionic liquids ${ }^{56}$ and phenyl-based molecules ${ }^{57}$ in CG simulations. These are only a few examples of the capability and flexibility of the Mie potential in modeling the interactions in the context of either atomistic or CG simulations.

\section{CASE STUDY: THE CARBON DIOXIDE MOLECULE}

A. Classical Intermolecular Potentials for $\mathrm{CO}_{2}$. Though clearly not the most complex molecular fluid, carbon dioxide has recently been in the spotlight as the urgent need to reduce its atmospheric concentration becomes evident. Many of the carbon capture abatement technologies that have been proposed require a knowledge of the thermodynamic equilibrium properties of the molecules at interfaces, be it in porous media (e.g., depleted reservoirs), in or in contact with complex fluids (absorption in liquid phases). In this context, molecular simulation can play a significant role in understanding and predicting the relevant physical phenomena. On the other hand, the modeling of interfacial systems requires the simulation of extremely large samples for long times.

Carbon dioxide has been widely studied using molecular simulation, and there are therefore a number of models have been developed. Here we present a brief overview of some of the semiempirical models that have been reported. The simplest conceivable model consists of a single spherical site with both repulsive and attractive contributions. The prototypical potential, $u$, of this type is the LJ potential, which incorporates a repulsive term, $u^{\text {rep }}$, and an attractive (dispersion) term, $u^{\text {att }}$, and is given by

$$
\begin{aligned}
u(r)^{\mathrm{LJ}}(r) & =4 \varepsilon\left[\left(\frac{\sigma}{r}\right)^{12}-\left(\frac{\sigma}{r}\right)^{6}\right] \\
& =u^{\mathrm{rep}}(r)+u^{\mathrm{att}}(r)
\end{aligned}
$$

where the size $\sigma$, and energy $\varepsilon$, parameters may be regressed from appropriate experimental data for different properties. Some common choices are based on the use of viscosity and diffusivity data, ${ }^{58-63}$ a direct fit of the critical temperature and density, $^{20,64,65}$ or on an overall representation of single-phase volumetric properties and fluid-phase equilibria data. ${ }^{66}$ Some of the available parametrizations for the $\mathrm{LJ}$ model of $\mathrm{CO}_{2}$ are summarized in Table 1. Clearly there is no unique set of values 
for the parameters, though the parameters obtained from critical properties invoking a corresponding states principle appear to provide the best overall performance. ${ }^{67}$ It is interesting to note that no matter what parameter set is chosen, the $\mathrm{LJ}$ model alone is unable to describe adequately the vapor-liquid equilibrium curve of $\mathrm{CO}_{2}$. In spite of the well-known fact that a simple single-site LJ interaction does not adequately represent the potential surfaces of real molecules, the model has been used extensively to simulate the fluid-phase equilibrium, ${ }^{66,68-73}$ transport properties, ${ }^{74-77}$ and the adsorption ${ }^{78,79}$ of supercritical $\mathrm{CO}_{2}$ and mixtures involving perfluoroalkanes. ${ }^{80}$ The single-site model is simple and useful but does not have the correct functional form to treat the uneven charge distribution of the carbon dioxide molecule.

Carbon dioxide is a small and rigid molecule, but the presence of significant electrostatic interactions makes the problem of its description far from trivial. Carbon dioxide is linear, with negligible bond bending and with electronegative centers at either end. Due to its symmetry, the molecule has no permanent dipole moment. However, the uneven charge distribution manifests itself in a significant quadrupole moment. In the more realistic potential models the effect of the quadrupole moment is incorporated, either by the incorporation of partially charged sites,

$$
u(r)=\left[u^{\mathrm{rep}}(r)+u^{\mathrm{att}}(r)\right]+\sum_{a} \sum_{b} \frac{q_{a} q_{b}}{4 \pi \varepsilon_{0} r_{a b}}
$$

where the sums are over all charged sites $(a, b)$ on the interacting molecules, or indirectly by assigning a centrally placed point quadrupole to the model,

$$
u=\left(u^{\mathrm{rep}}+u^{\mathrm{att}}\right)+u^{\mu \mu}+u^{\mu \mathrm{Q}}+u^{\mathrm{QQ}}+\ldots
$$

In eq $6, q_{a}$ is the partial charge of site $a, r_{a b}$ is the center-center distance between charged sites $a$ and $b, \varepsilon_{0}$ is the vacuum permittivity, and in eq 7 the superscripts $\mu \mu, \mu \mathrm{Q}$ and QQ refer to the dipole-dipole, dipole-quadrupole, and quadrupolequadrupole interactions, respectively, which are all included for completeness. For an axially symmetric molecule such as $\mathrm{CO}_{2}$, the corresponding quadrupole-quadrupole energy $u^{\mathrm{QQ}}$ can be obtained from a multipole expansion as $58,81,82$

$$
u^{\mathrm{QQ}}=\frac{3 Q^{2}}{4 r^{5}} f_{\Omega}
$$

where $f_{\Omega}$ is a function of the relative molecular orientation. ${ }^{58}$

The simplest of these quadrupolar models would consist of a $\mathrm{LJ}$ (or other similar) spherical interaction, with a centrally placed point quadrupole; the sum of eqs 5 and 8 . This potential has been used by Nouacer and Shing ${ }^{83}$ in grand canonical Monte Carlo simulations of naphthalene and $\mathrm{CO}_{2}$. The study included an analysis of entrainment with water modeled as a LJ sphere with a point dipole.

As far back as 1974 Gibbons and Klein ${ }^{52}$ proposed a twocenter model to study the thermodynamic properties of $\mathrm{CO}_{2}$ in the solid state. The sites were taken to interact through a Mie potential, and the exponents $\lambda_{\mathrm{r}}=9$ and $\lambda_{\mathrm{a}}=6$ were found to give the best description of the thermophysical and elastic properties. Johnson and Shaw ${ }^{84}$ later proposed a two-center model with force centers on the oxygen atoms (the carbon atom is ignored in their model), using an exponential-6 (exp-6, as opposed to Mie) potential to represent the repulsive and dispersive interactions between the spherical sites. This model may be spherically averaged, either with an exp-6 or in tabulated form. ${ }^{85}$ Möller and Fischer ${ }^{86}$ have proposed a fused model comprising two overlapping LJ spheres (where the centers of the spheres do not correspond to any particular atom center) with an embedded central point quadrupole as in eq 8 . Four adjustable parameters were used to characterize the model, because now the bond length and the square of the quadrupolar moment $Q^{* 2}=Q^{2} / \varepsilon \sigma^{5}$ are also estimated by comparison to experimental volumetric properties. The potential provides an appropriate model of the vapor-liquid equilibria (VLE), ${ }^{87,88}$ supercritical properties, ${ }^{89-91}$ adsorption on carbon pores ${ }^{92}$ and nanotubes, ${ }^{93}$ and even second derivative thermodynamic properties such as the Joule-Thomson inversion curves. $^{94-96}$ This two-site LJ plus quadrupole model has been used extensively to model a number of binary ${ }^{97}$ and ternary mixtures ${ }^{98}$ comprising $\mathrm{CO}_{2}$. Elongated molecules represented with Kihara potentials have also been used to model the repulsive-dispersive part of the potential. After decoration with a suitable central quadrupole, ${ }^{99}$ this model is found to provide a good description of the vapor-liquid behavior of $\mathrm{CO}_{2}$.

For a more detailed description one should in principle treat the three distinct atoms present in the molecule. Three fused LJ spheres may be used as a repulsive-dispersive nonspherical core, with an additional point quadrupole (3CLJQ). ${ }^{100-102}$ Murthy et al. ${ }^{100}$ proposed and compared several two- (2CLJQ) and three-site (3CLJQ) models with a centrally placed quadrupole moment, pointing out the superiority of a three-site model with distinct size and energy parameters for the spheres representing the carbon and the two oxygen atoms. A refined version of the 3CLJQ model has been developed recently by Merker et al. ${ }^{102}$ to describe the VLE of $\mathrm{CO}_{2}$. The aim of their study was to represent the VLE with a better description of the molecular structure. The 3CLJQ model of Merker et al. is found to provide an accurate description of the saturation densities and vapor pressure and is also able to reproduce shear viscosity and thermal conductivity data. A different treatment of the quadrupole moment in the $\mathrm{CO}_{2}$ molecules was made in the elementary physical model (EPM) of Harris and Yung. ${ }^{103}$ Their model comprises three LJ spheres with partial charges associated with each site (instead of a central point quadrupole) positioned to reproduce a net quadrupole moment of $Q=-4.3 \times 10^{-26} \mathrm{esu}\left(-1.43 \times 10^{-39} \mathrm{C} \mathrm{m}^{2}\right)$. In a readjustment of the parameters they proposed a refined model, referred to as EPM2, with better agreement in the critical region. Models with flexible bond angles have also been considered, but no significant improvement over the other models is found. Several other parametrizations of the EPM2 model are available depending on the particular property sought. ${ }^{104-110} \mathrm{~A}$ variant of this potential with an exp-6 core instead of LJ spheres has also been proposed. ${ }^{111}$ These three-center plus electrostatic charge models are comparatively detailed with respect to both the geometry of the molecule and the potential energy surface and have been successfully used to study the fluid structure, ${ }^{112}$ vaporliquid equilibria, ${ }^{105,113-116}$ transport properties, ${ }^{105}$ interfacial kinetics, ${ }^{117}$ solvation properties, ${ }^{118}$ selective adsorption of $\mathrm{CO}_{2}$ on activated carbons ${ }^{119}$ and templated nanomaterials, ${ }^{120}$ and the solubility in supercritical $\mathrm{CO}_{2},{ }^{121-125}$ amongst other work. They are considered to be the de facto standard for simulations of the fluid and solid phases of $\mathrm{CO}_{2}$. A related three-center polarizable rigid model has recently been reported by Persson, ${ }^{126}$ comprising sites interacting through a modified Buckingham exp- 6 potential with an anisotropic (three-body) Axilrod-Teller dispersion correction, and Gaussian charge densities localized on the atomic sites. In this model the experimental quadrupole moment, polarizability, and bond distances are used during the parametrization. In general, the second and third virial coefficients are underestimated 
with simple pairwise interaction models of $\mathrm{CO}_{2}$, while the model of Persson reproduces these properties with good accuracy.

More sophisticated multiparameter potentials are available, usually developed to reproduce volumetric data and distribution functions obtained from neutron scattering experiments. ${ }^{127}$ Ultimately, as mentioned earlier, one may obtain the information about the intermolecular potential directly from quantum-mechanical calculations. ${ }^{102,128-132}$ A notable example of this type of approach is the potential that has been suggested by Tsuzuki et al., ${ }^{133,134}$ who presented the results of $a b$ initio calculations using a three-site LJ model with partial charges. Unlike the EPM-type models, the partial charges do not coincide with the LJ centers. In general, the gas-phase potential energy surfaces obtained using $a b$ initio methods are not able to reproduce the properties of condensed phases. These potentials are usually tested by computing the second virial coefficient. To use these potentials in molecular simulation of fluid phases, it is necessary to modify the $a b$ initio potential energy surfaces. Merker et al. ${ }^{102}$ followed this approach to obtain their 3CLJQ model. The initial locations of the LJ sites were the same as the positions of the nuclei computed using a Hartree-Fock level of theory, while the magnitude of the point quadrupole located at the center of the molecule was calculated by placing a single $\mathrm{CO}_{2}$ molecule into a dielectric cavity to approximate the liquid-like behavior using the Møller-Plesset 2 method. These parameters were subsequently adjusted to reproduce the liquid density, vapor pressure, and enthalpy of vaporization.

B. Coarse-Graining $\mathrm{CO}_{2}$ as a Single-Site Model. In a molecular dynamics simulations, the complexity of the potential model has a direct effect on the computational effort that is required. To evaluate the interaction energy between two carbon dioxide molecules interacting via the EPM model, for example, one must calculate $3^{2}=9$ site - site distances for each pair of molecular interactions and, due to the nonsphericity, solve for the angular momentum conservation equations at each time step. Additionally, the presence of point charges requires special computational techniques, e.g., the use of the Ewald summation, ${ }^{135-137}$ reaction-field, ${ }^{138}$ or Wolf ${ }^{139,140}$ methods, to account for the long-range interactions. Assuming that fluid-phase equilibria are dominated by the energetic contributions to the interactions rather than by specific molecular shape, ${ }^{19}$ one can simplify the intermolecular potential by considering that a spherical geometry will be adequate for most calculations, particularly for states of moderate density. The use of a single spherical interaction site model can decrease the computational effort by at least an order of magnitude of CPU time.

In general, multipolar interactions are angle dependent (cf. eq 8), so one must specify both center-to-center distances and relative orientations for a proper evaluation of the intermolecular potential. When one performs an appropriate Boltzmann or free-energy angle average, ${ }^{19,58}$ an angle-independent potential function is obtained, which can be used in corresponding states correlations to obtain a simple isotropic multipolar potential (IMP), including angle-average contributions of the type

$$
\begin{aligned}
& u^{\mu \mu}(r) \approx-\frac{\beta \mu^{4}}{3 r^{6}} \\
& u^{\mu \mathrm{Q}}(r) \approx-\frac{\beta \mu^{2} Q^{2}}{r^{8}} \\
& u^{\mathrm{QQ}}(r) \approx-\frac{7 \beta Q^{4}}{5 r^{10}}
\end{aligned}
$$

The resulting overall interaction potential, the sum of eq 5 and eqs 9-11, is isotropic; i.e., it only depends on the radial intermolecular distance $r$. The IMP model is a function of temperature $T$, because $\beta=$ $1 / k_{\mathrm{B}} T$, and is thus not a true potential, but rather a potential of mean force (free energy) corresponding to an "effective" force field. For isothermal simulations this does not present a problem, though the potential will depend on the thermal state being simulated.

In representing $\mathrm{CO}_{2}$ with the original IMP parametrization, a constant value of the quadrupole moment $Q=-4.1 \times 10^{-26}$ $\operatorname{esu}^{141}\left(-1.367 \times 10^{-39} \mathrm{C} \mathrm{m}^{2}\right)$ is used, with an energy $\varepsilon$ / $k_{\mathrm{B}}=215 \mathrm{~K}$ and diameter $\sigma=3.748 \AA^{19}{ }^{19}$ The critical temperature obtained from simulations of the IMP fluid using finite-size scaling calculations is $304.8 \pm 0.5 \mathrm{~K},{ }^{20}$ which compares favorably with the experimental values of $304.21 \mathrm{~K}^{142,143} \mathrm{It}$ is feasible that one could obtain different parameter values for the IMP model that would provide a better description of other properties, such as the coexistence densities, vapor pressures, etc. ${ }^{19,22}$ Alternatively, the value of the quadrupole moment could be varied, using it as an adjustable parameter to provide a better representation of a given property.

A question immediately arises from the discussion of the previous section: to which extent can one represent the thermodynamic properties of $\mathrm{CO}_{2}$ with a state independent single-site spherical intermolecular potential? This may at first sight appear too crude an approximation because it is wellknown that simple spherically symmetric intermolecular potentials such as the LJ model cannot be used to capture the fluid-phase equilibria of $\mathrm{CO}_{2}$ with reasonable accuracy ${ }^{20,22}$ (cf. section III'A). To assess the possibility of simplifying the description of $\mathrm{CO}_{2}$ with a single spherical core, we propose a new "top-down" coarse-graining approach based on the use of the SAFT-VR EoS for molecules comprising Mie segments (cf. Appendix A1). ${ }^{34}$ By making use of an analytical SAFT-VR free energy, which is based on an explicit intermolecular potential model, one can rapidly explore a very large parameter space and estimate the parameters that provide the best representation of the available macroscopic experimental data. The approach also allows one to assess the importance or otherwise of explicitly treating the nonsphericity of $\mathrm{CO}_{2}$ by simply comparing the representation for different numbers of interactions sites, cf. the number of molecular segments $m_{\mathrm{s}}$ in the SAFT-VR treatment (see Appendix A1). We opt for a good overall description of fluid-phase equilibria over the entire vapor-liquid temperature range as the most important characteristic of our coarse-grained model. To this end the model parameter values are estimated by optimizing the SAFT-VR Mie description for a set of experimental data over a range of subcritical temperatures: the vapor pressure and saturated liquid density are considered in this particular case. It should be emphasized that other properties such as the heat capacity of the liquid, Joule-Thomson inversion curve, or speed of sound of the fluid could also have been taken into account. These other properties were not considered in developing the potential model, however, because the equation of state is based on a high-temperature perturbation theory, and the calculation of second-derivative properties with respect to temperature can lead to some discrepancies with the "exact" values as determined from molecular simulation, particularly at low temperature. $^{144}$ We advocate the use of a simple estimation procedure that involves only first-derivatives of the Helmholtz free energy function (the pressure and chemical potential corresponding to phase equilibria) by minimizing the relative 
Table 2. SAFT- $\gamma$ Mie Force Field Parameters for $\mathrm{CO}_{2}$

\begin{tabular}{|c|c|c|c|c|}
\hline$\left(\varepsilon / k_{\mathrm{B}}\right) / \mathrm{K}$ & $\sigma / \AA$ & $\lambda_{\mathrm{r}}$ & $\lambda_{\mathrm{a}}$ & comment \\
\hline 361.69 & 3.741 & 23.0 & 6.66 & original optimization $^{a}$ \\
\hline 353.55 & 3.741 & 23.0 & 6.66 & $\begin{array}{l}\text { rescaled to match } \\
\text { the critical temperature } \\
\text { and interfacial tension }\end{array}$ \\
\hline
\end{tabular}

${ }^{a}$ These parameters are obtained using our SAFT- $\gamma$ Mie EoS and the objective function given by eq 12 . The AAD\%s calculated using this approach are $0.69 \%$ for the saturated liquid density, and $5.84 \%$ for the vapor pressure, respectively, over the temperature range from 228 to $273 \mathrm{~K}$.

residuals between the measured and estimated vapor pressures $P^{\text {sat }}$ and saturated liquid densities $\rho^{\mathrm{L}}$, as a function of temperature.

If one assumes that these properties of $\mathrm{CO}_{2}$ can be represented with a single Mie interaction site $\left(m_{\mathrm{s}}=1\right)$, the objective function $F$ can written as follows:

$$
\begin{aligned}
\min _{\sigma, \varepsilon, \lambda_{r}, \lambda_{a}} F\left(\sigma, \varepsilon, \lambda_{\mathrm{r}}, \lambda_{\mathrm{a}}\right)= & \min _{\sigma, \varepsilon, \lambda_{\mathrm{r}}, \lambda_{\mathrm{a}}}\left[\sum_{i=1}^{N_{\mathrm{p}}}\left(\frac{P_{i}^{\mathrm{sat}}\left(T ; \sigma, \varepsilon, \lambda_{\mathrm{r}}, \lambda_{\mathrm{a}}\right)-P_{i}^{\mathrm{sat}, \exp }(T)}{P_{i}^{\mathrm{sat}, \exp }(T)}\right)^{2}\right. \\
& \left.+\sum_{j=1}^{N_{\mathrm{d}}}\left(\frac{\rho_{j}^{\mathrm{L}}\left(T ; \sigma, \varepsilon, \lambda_{\mathrm{r}}, \lambda_{\mathrm{a}}\right)-\rho_{j}^{\mathrm{L}, \exp }(T)}{\rho_{j}^{\mathrm{L}, \exp }(T)}\right)^{2}\right]
\end{aligned}
$$

where $N_{\mathrm{p}}$ and $N_{\mathrm{d}}$ are the number of experimental points for the vapor pressure and saturated liquid densities, respectively. Note that $F$ is a function of the four interaction parameters of the Mie potential including the repulsive and attractive exponents $\lambda_{\mathrm{r}}$ and $\lambda_{\mathrm{a}}$. A common consideration with the attractive exponent is to fix it to the LJ value of $\lambda_{a}=6$ to follow the London law for the forces. However, London forces invoke a simple dispersion attraction without permanent multipoles, which is not the case for $\mathrm{CO}_{2}$. Both exponents are therefore optimized here to capture effectively the unique multipolar interactions present. The intermolecular SAFT $-\gamma$ model parameters are obtained by optimizing the theoretical description of the experimental fluidphase equilibrium; a Levenberg-Marquardt algorithm ${ }^{145}$ is used to minimize the objective function, F. As is common practice with equation of state parameters, experimental data for the vapor pressure and saturated liquid density are used in the determination of potential parameters; in this case we used 40 state points (temperature, pressure, density) equally spaced in temperature from the triple point to $90 \%$ of the critical point $T_{c}$, i.e., $T / T_{c}=0.9$. Smoothed experimental data were taken directly from the NIST database. ${ }^{143}$ It should be pointed out that the proposed approach will result in a slight overestimate of the critical temperature and pressure predicted by the equation of state. Such a behavior is unavoidable with any algebraic EoS unless a specific treatment of the near-critical region is made. ${ }^{146}$ This does not mean that the resulting intermolecular potential will lead to a poor estimate of the critical point by simulation, as we will show later in the paper. We obtain the following optimal molecular parameters for our single-site SAFT- $\gamma$ Mie force field for $\mathrm{CO}_{2}: \sigma=3.741 \AA, \varepsilon / k_{\mathrm{B}}=361.69 \mathrm{~K}, \lambda_{\mathrm{a}}=6.66$, and $\lambda_{\mathrm{r}}=23.0$. These molecular parameters are also presented in Table 2 for ease of comparison. A single-site Mie potential provides an accurate description of both the vapor pressure and saturated liquid density. Details of the property predictions with this new
SAFT- $\gamma$ intermolecular potential model for $\mathrm{CO}_{2}$ are described in section IV.

C. Molecular Simulation Details. The fluid-phase equilibria and second-derivative properties of the SAFT- $\gamma$ Mie CG model of $\mathrm{CO}_{2}$ are determined using Monte Carlo simulation of the fluid in the grand canonical (GC-MC) and isobaric-isothermal (NPT-MC) ensembles, respectively. The simulation of phase equilibria is not straightforward, as the system will form an interface between coexisting phases, with a free energy that is higher than that of the coexisting states; this energetic barrier has to be overcome for a correct description of the coexistence properties with the GC-MC technique. To overcome the interfacial free energy barrier during the GC-MC simulation, we make use of the so-called multicanonical methods ${ }^{147,148}$ that modify the acceptance criteria using a preweighting distribution function, which allows for a uniform sampling of all states without being trapped in energy minima for temperatures below the critical point. ${ }^{149}$ The aforementioned preweighting function is not known a priori and different methods have been proposed for its evaluation. ${ }^{149}$ In our work we use the transition matrix Monte Carlo method, ${ }^{150-152}$ as implemented by Errington, ${ }^{153,154}$ to calculate the probability distribution $\Pi(N ; \mu, V, T)$ for numbers of particles ranging from $N=0$ to $N_{\text {max }}$ in a self-consistent way using the acceptance probability between the microstates sampled during the simulation. To calculate $\Pi(N ; \mu, V, T)$, all of the information about the microstates, including those that are rejected, is taken into account, which makes the method very efficient. During the simulation $\Pi(N ; \mu, V, T)$ can be used to obtain the preweighted distribution that modifies the acceptance probability of the GC-MC method to access the low-probability mixedphase states using the multicanonical method. It should be stressed that even when the simulation is biased using the multicanonical method, $\Pi(N ; \mu, V, T)$ has to be calculated using the unbiased acceptance probability. Once the simulation has been undertaken, one can make use of the histrogram reweighting (HR) technique $^{155,156}$ to determine the chemical potential at coexistence, $\mu_{\text {coex }}(T)$. To reweight the data at a single temperature, the following expression can be used:

$$
\ln \Pi(N ; \mu, V, T)=\ln \Pi\left(N ; \mu_{0}, V, T\right)+\beta\left(\mu-\mu_{0}\right) N
$$

where the chemical potential $\mu$ is tuned until the areas underneath the two peaks of the bimodal number distribution $\Pi(N ; \mu, V, T)$ are equal, corresponding to the coexisting vapor and liquid phases. In eq 13 the subscript 0 refers to that of the original simulated state. In practice, it is convenient to have an estimate of $\mu_{\text {coex }}(T)$ to start the simulations; as shown by Errington, ${ }^{153,154}$ any suitable value of $\mu$ will in principle lead to similar results after reweighting. A good estimate can be obtained by running a set of simulations for a small system at any value of $\mu$, and then reweighting the data. This approach provides an estimate of $\mu_{\text {coex }}(T)$ that is no more than $1 \%$ of that of the large systems. If the low-density limit $N=0$ has been sampled, the vapor pressure can be calculated by using the ideal gas as a reference state: ${ }^{153,154}$

$$
\beta P V=\ln \left[\sum_{N} \Pi\left(N ; \mu_{\text {coex }}, V, T\right)\right]-\ln \Pi\left(0 ; \mu_{\text {coex }}, V, T\right)-\ln 2
$$

Finally, the surface tension can be estimated using the finitesize scaling (FSS) formalism of Binder, ${ }^{157}$ which can be 
expressed as

$$
\beta \gamma_{L}=\frac{A_{L}}{2 L^{2}}=c_{1} \frac{1}{L^{2}}+c_{2} \frac{\ln L}{L^{2}}+\beta \gamma
$$

where $\gamma_{L}$ is the surface tension of the finite system, $\gamma$ is the surface tension of the infinite system, and $L$ is the length of the cubic simulation box; the factor of a one-half is included because the system will exhibit two interfaces. Using this approach, it is possible to extrapolate $\gamma$ from a series of simulations for systems of different sizes. ${ }^{158}$ The interfacial free energy $A_{L}$ is obtained from the particle number distribution $\Pi\left(N ; \mu_{\text {coex }} V, T\right)$ as

$$
\begin{aligned}
\beta A_{L}= & \frac{1}{2}\left\{\max \left[\ln \Pi\left(N ; \mu_{\text {coex }}, V, T\right)\right]_{\text {liq }}\right. \\
& \left.+\max \left[\ln \Pi\left(N ; \mu_{\text {coex }}, V, T\right)\right]_{\text {vap }}\right\} \\
& \left.-\min \left[\ln \Pi\left(N ; \mu_{\text {coex }}, V, T\right)\right]_{\mathrm{d}}\right\}
\end{aligned}
$$

where $\max \left[\ln \Pi\left(N ; \mu_{\text {coex }} V, T\right)\right]_{\text {liq }}$ and $\max \left[\ln \Pi\left(N ; \mu_{\text {coex }} V, T\right)\right]_{\text {vap }}$ correspond to the maximum of the logarithm of the particle number probability for the liquid and vapor peaks, respectively, and $\min \left[\ln \Pi\left(N ; \mu_{\text {coex }} V, T\right)\right]_{\mathrm{d}}$ corresponds to the minimum between the liquid and vapor domains. A test-area $\mathrm{MC}$ technique $^{159}$ could also have been used in this case, but we opted for the FSS method as this can also be used to provide an accurate estimate of the critical point.

The calculations of volumetric and second-derivative thermodynamic properties are carried out using NPT-MC simulations, where one can determine the coefficient of thermal expansion $\alpha_{P}$, the isothermal compressibility $\kappa_{T}$, and the configurational heat capacity at constant pressure $C_{P}^{\text {conf }}$ directly. These properties are estimated by using the appropriate expressions involving the averages of the fluctuations of the configurational internal energy $U^{\text {conf }}$, the configurational enthalpy $H^{\text {conf }}$, the volume $V$, and their combinations. These expressions are given by $95,160,161$

$$
\begin{aligned}
& \alpha_{P}= \frac{1}{\langle V\rangle}\left(\frac{\partial\langle V\rangle}{\partial T}\right)_{P} \\
&= \frac{1}{\langle V\rangle k_{\mathrm{B}} T^{2}}\left(\left\langle V H^{\mathrm{conf}}\right\rangle-\langle V\rangle\left\langle H^{\mathrm{conf}}\right\rangle\right) \\
& \kappa_{T}=-\frac{1}{\langle V\rangle}\left(\frac{\partial\langle V\rangle}{\partial P}\right)_{T}=\frac{1}{\langle V\rangle k_{\mathrm{B}} T}\left(\left\langle V^{2}\right\rangle-\langle V\rangle^{2}\right) \\
& C_{P}^{\text {conf }}=\left(\frac{\partial U^{\text {conf }}}{\partial T}\right)_{P}+P\left(\frac{\partial\langle V\rangle}{\partial T}\right)_{P}-N k_{\mathrm{B}} \\
&=\frac{1}{k_{\mathrm{B}} T^{2}}\left(\left\langle U^{\text {conf }} H^{\text {conf }}\right\rangle-\left\langle U^{\text {conf }}\right\rangle\left\langle H^{\text {conf }}\right\rangle\right) \\
&+\frac{1}{k_{\mathrm{B}} T^{2}}\left(\left\langle V H^{\text {conf }}\right\rangle-\langle V\rangle\left\langle H^{\text {conf }}\right\rangle\right)-N k_{\mathrm{B}}
\end{aligned}
$$

where the \langle\rangle brackets correspond to ensemble averages. To make a comparison with experimental data, the ideal contribution $C_{P}^{\text {id }}$ to the heat capacity at constant pressure is added to the configurational part,

$$
C_{P}=C_{P}^{\text {id }}+C_{P}^{\text {conf }}
$$

where the ideal contribution is taken from experimental correlations. ${ }^{142,143}$ Once these properties have been calculated, the other second-derivative properties can be obtained using the standard thermodynamic relationships. The isochoric heat capacity $C_{V}$, the Joule-Thomson coefficient $\mu_{\mathrm{JT}}$, and the speed of sound $\omega$ can be conveniently obtained from ${ }^{162}$

$$
\begin{gathered}
C_{P}-C_{V}=T\langle V\rangle \frac{\alpha_{P}^{2}}{\kappa_{T}} \\
\mu_{\mathrm{JT}}=\frac{\langle V\rangle}{C_{P}}\left[T \alpha_{P}-1\right] \\
\omega^{2}=\frac{C_{P}}{C_{V}} \frac{\langle V\rangle}{\kappa_{T}} \frac{M_{\mathrm{w}} N_{\mathrm{A}}}{N}
\end{gathered}
$$

where $M_{\mathrm{w}}$ and $N_{\mathrm{A}}$ are the molecular weight and the Avogadro constant, respectively.

The calculations of the fluid-phase equilibria using GC-MC simulation are carried out in a cubic simulation box of volume $L^{3}$ with $L^{*}=L / \sigma=14$. An estimate of $\mu_{\text {coex }}$ is obtained using a small system of length size $L^{*}=6$ and a HR technique is used to locate the coexistence point. Simulation runs are carried out for $1 \times 10^{9}$ MC configurations for the system with $L^{*}=6$ and about $(4-5) \times 10^{9}$ for the system with $L^{*}=14$. In all cases, the cutoff of the potential is taken to be half of the simulation box length, $R_{\mathrm{c}}=0.5 L^{*}$, and standard long-range corrections to the energy and pressure virial are included. ${ }^{135,136}$ Fixed probabilities of $70 \%$ for the insertion-deletion attempts and $30 \%$ for the particle displacements are chosen. Extra simulations involving systems with sizes corresponding to $L^{*}=8,10$, and 12 are also carried out to allow for an extrapolation to the macroscopic interfacial tension. The critical temperature $T_{\mathrm{c}}$ is estimated using a Wegner expansion up to the first-order correction term: ${ }^{163-165}$

$$
\rho_{\mathrm{l}}-\rho_{\mathrm{v}}=B_{0}|\tau|^{\beta_{\mathrm{c}}}+B_{1}|\tau|^{\beta_{\mathrm{c}}+\Delta}
$$

where $\rho_{\mathrm{l}}$ and $\rho_{\mathrm{v}}$ correspond to the coexisting liquid and vapor densities, respectively, $\tau=1-T / T_{\mathrm{c}}, \beta_{\mathrm{c}}=0.325$ is the critical exponent that is fixed at its universal renormalization-group value, $\Delta$ is the so-called gap exponent that is taken as 0.51 , and $B_{i}$ are the correction amplitudes. The critical density $\rho_{c}$ is calculated by means of a least-squares fit of the rectilinear diameter law:

$$
\frac{\rho_{\mathrm{l}}+\rho_{\mathrm{v}}}{2}=\rho_{\mathrm{c}}+D|\tau|
$$

where $D$ is the correlation parameter. A more accurate description for the critical region is possible with, e.g., FSS techniques, ${ }^{149,166,167}$ but as the scope of the present work is not an exhaustive treatment of the critical region, we have limited ourselves to the use of the simple scaling relations embodied in eqs 24 and 25 . The critical pressure is obtained by extrapolation using the Clausius-Clapeyron equation:

$$
\ln P=C_{1}+\frac{C_{2}}{T}
$$

where $C_{1}$ and $C_{2}$ are correlation parameters.

The NPT-MC simulations are carried out for a system of $N=$ 800 particles. The runs are performed for $7.5 \times 10^{4} \mathrm{MC}$ cycles for equilibration and $2.5 \times 10^{5}$ cycles to accumulate the averages. In our simulations, one NPT-MC cycle refers to $N$ Monte Carlo steps, 5\% of which corresponds to an attempt to change the volume of the system and $95 \%$ to attempted displacements of the particles. The cutoff of the potential is fixed to $R_{\mathrm{c}}=4 \sigma$ in the NPT simulations, and standard longrange corrections to the energy and pressure virial are also 


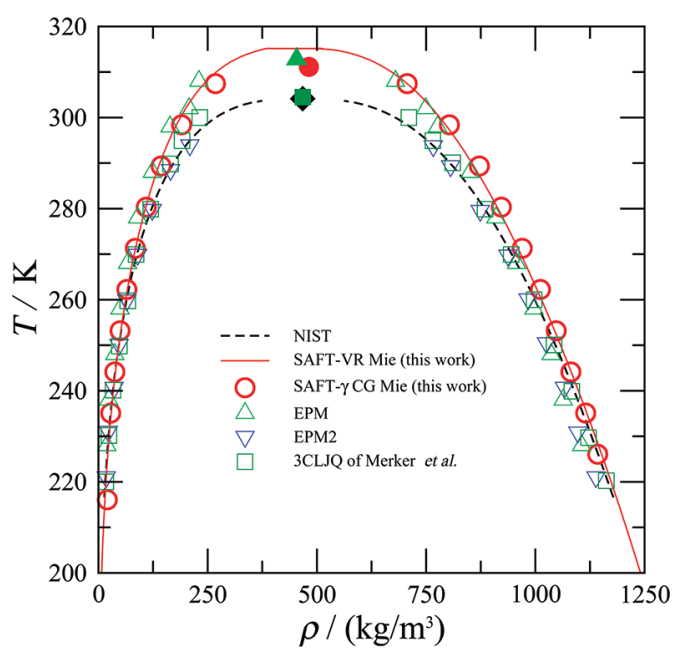

Figure 1. Temperature-density vapor-liquid coexistence curve for $\mathrm{CO}_{2}$. The dashed curve denotes the smoothed experimental data from NIST, ${ }^{142,143}$ the continuous curve the SAFT-VR Mie EoS (this work), the circles are obtained by Monte Carlo simulation for the SAFT- $\gamma$ CG Mie model of $\mathrm{CO}_{2}$ (this work), the up and down triangles represent the simulation results for the EPM and EPM2 models, ${ }^{103}$ respectively, and the squares represent the results for the 3CLJQ model of Merker et al. ${ }^{102}$ The filled diamond represents the experimental critical point, ${ }^{142,143}$ the filled circle the critical point obtained for our model, the filled triangle the critical point of the EPM model, and the filled square the critical point of the 3CLJQ model of Merker et al.

included. ${ }^{135,136}$ Uncertainties for the different properties are obtained by performing three independent runs for both the GC-MC and NPT-MC simulations.

\section{RESULTS}

The results of the GC-MC simulations for the coexistence curve and vapor pressure of our SAFT- $\gamma$ CG single-center Mie model of $\mathrm{CO}_{2}$ are presented in Figures 1 and 2. The simulation data for our new model are compared with the calculations obtained with the SAFT-VR Mie EoS (which is used to develop the potential model), ${ }^{34}$ the corresponding data obtained for the EPM and EPM2 models of Harris and Yung ${ }^{103}$ and for the 3CLJQ model of Merker et al., ${ }^{102}$ and with the available experimental data. ${ }^{142,143}$ As can observed from Figure 1, the simulation data for the SAFT- $\gamma$ model and theory are in good agreement with the experimental vapor-liquid coexistence envelope, except close to the critical point, where a small overestimate is expected with the theory; one should appreciate that the GC-MC technique will also lead to a slight overestimate of the critical temperature unless a full FSS treatment is made. The average absolute deviation (AAD\%) ${ }^{168}$ obtained with our model for the coexistence liquid density is $2.6 \%$ for a temperature range between 228 and $289 \mathrm{~K}$, compared with $2.0 \%$ and $1.7 \%$ obtained with the EPM and EPM2 models, respectively, over the same range. The 3 CLJQ model of Merker et al. reproduces the experimental data very well with an $\mathrm{AAD} \%$ of $4 \%$ for the larger temperature range of $220-300 \mathrm{~K}$. Our model predicts the vapor density with an accuracy of $6 \%$ for temperature between 228 and $289 \mathrm{~K}$. By comparison, the EPM and EPM2 models overpredicts this property by more than $10 \%$, while the 3 CLJQ model of Merker et al. underpredicts the vapor density by $5 \%$.

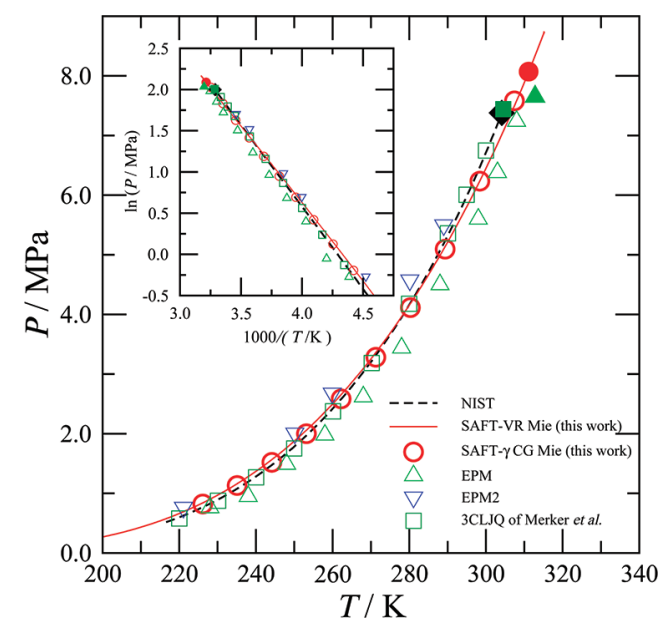

Figure 2. Vapor pressure for $\mathrm{CO}_{2}$. The dashed curve denotes the smoothed experimental data from NIST, ${ }^{142,143}$ the continuous curve the SAFT-VR Mie EoS, the circles are obtained by Monte Carlo simulation for the SAFT- $\gamma$ CG Mie model of $\mathrm{CO}_{2}$ (this work), the up and down triangles represent the simulation results for the EPM and EPM2 models, ${ }^{103}$ respectively, and the squares represent the results for the 3CLJQ model of Merker et al. ${ }^{102}$ The filled diamond represents the experimental critical point, ${ }^{142,143}$ the filled circle the critical point obtained for our model, the filled triangle the critical point of the EPM model, and the filled square the critical point of the 3CLJQ model of Merker et al.

The critical point for the SAFT- $\gamma$ CG Mie model of $\mathrm{CO}_{2}$ corresponds to a critical temperature of $T_{\mathrm{c}}=311.13 \mathrm{~K}$ compared with the experimental value of $304.128 \mathrm{~K}$, which is an overprediction of $2.3 \%$. By comparison, a critical temperature $T_{\mathrm{c}}=$ $312.8 \mathrm{~K}$ is obtained with the EPM model, which is about $3 \%$ higher than the experimental value. The EPM2 model provides a correct description of the critical point, because it has been parametrized for that purpose. Our model can also be compared with the more sophisticated models for $\mathrm{CO}_{2}$, such as the twocenter LJ plus point quadrupole (2CLJQ) model of Möller and Fischer, ${ }^{86}$ which was parametrized using saturation properties of the fluid. This model predicts a critical temperature of $T_{c}=$ $307.83 \mathrm{~K}$, which corresponds to an overprediction of about $1.2 \%$. The 3CLJQ model developed for $\mathrm{CO}_{2}$ by Merker et al. ${ }^{102}$ describes the critical region with very good accuracy.

From Figure 2 it is also apparent that our SAFT- $\gamma$ CG Mie model reproduces the experimental vapor-pressure data for $\mathrm{CO}_{2}$ very well, corresponding to an $\mathrm{AAD} \%$ of $2.9 \%$ for temperatures ranging between 228 and $298 \mathrm{~K}$. The EPM and EPM2 models both give rise to an significant deviation of the vapor pressure of more than $10 \%$, while the model of Zhang and Duan ${ }^{107}$ leads to an overprediction of about $15 \%$. For the 3CLJQ model of Merker et al. ${ }^{102}$ a deviation of only $1.8 \%$ for the saturation pressure is obtained. The good description of the experimental vapor pressure with our model is perhaps not that surprising, because it is well-known that the variable repulsive and dispersive exponents of the Mie potential are the key feature which allow for this property to be captured accurately. ${ }^{33}$ The same observation has been made by Potoff and Bernard-Brunel ${ }^{49}$ in their simulation studies on the use of the Mie potential to develop united atom models of the alkanes and perfluoroalkanes. Though one could improve the description of the fluid-phase behavior of $\mathrm{CO}_{2}$ by further refinement of our CG Mie model, it is important 


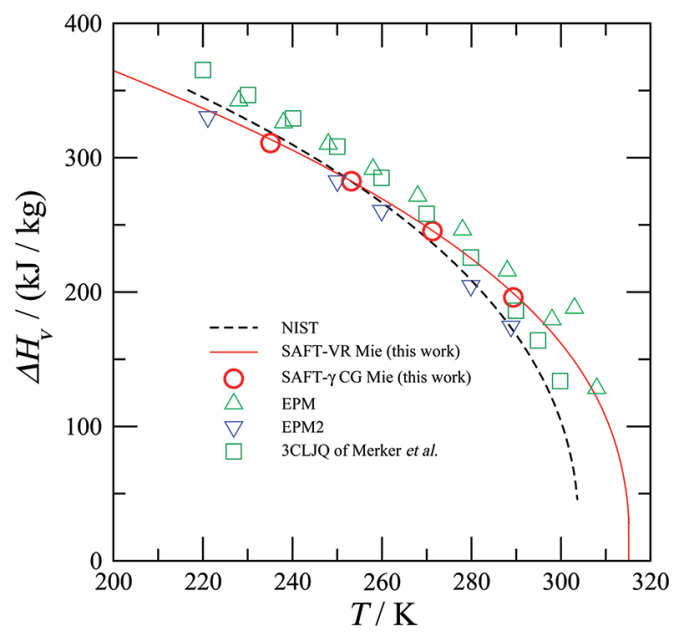

Figure 3. Vaporization enthalpy $\Delta H_{\mathrm{v}}$ as a function of the temperature for $\mathrm{CO}_{2}$. The dashed curve denotes smoothed experimental data from NIST, ${ }^{142,143}$ the continuous curve the SAFT-VR Mie EoS (this work), the circles are the results obtained by Monte Carlo simulation for the SAFT- $\gamma$ CG Mie model of $\mathrm{CO}_{2}$ (this work), the up and down triangles represent the simulation results for the EPM and EPM2 models, ${ }^{103}$ respectively, and the squares represent the results for the 3CLJQ model of Merker et al. ${ }^{102}$

to stress that the values of the interaction parameters are obtained with the SAFT-VR Mie $\operatorname{EoS}^{34}$ without any a posteriori adjustment of the CG model. It is also very gratifying to find that a single-site CG model can be used to reproduce the phase behavior of $\mathrm{CO}_{2}$ with good accuracy, as long as the softness/ hardness of the interaction is modeled appropriately.

The SAFT- $\gamma$ CG Mie model of $\mathrm{CO}_{2}$ is obtained using the SAFT-VR EoS by estimating the intermolecular parameters solely to the saturation properties of the fluid. The prediction of other thermodynamic properties, not used in the parametrization of the model, provides a stringent assessment of the robustness of our single-site model. For example, our model provides a good prediction of the enthalpy of vaporization $\Delta H_{\mathrm{v}}$ as can be observed in Figure 3, which corresponds to an AAD\% of about 5\% for this caloric property. The EPM and EPM2 predict $\Delta H_{\mathrm{v}}$ with $\mathrm{AAD} \%$ s of about $9 \%$ and $2 \%$, respectively. The corresponding $\mathrm{AAD} \%$ obtained with the model of Merker et al. ${ }^{102}$ is $8.1 \%$ for this property over the temperature range from 200 to $300 \mathrm{~K}$. It is important to reiterate that as shown in Figures $1-3$, the simulated properties for the SAFT- $\gamma$ CG Mie model are in excellent agreement with the predictions of the SAFT-VR EoS, which is why it is possible to use the theory to estimate the intermolecular parameters in an accurate manner for a very broad range of thermodynamic states.

We have also determined the interfacial tension of the vapor-liquid interface of our $\mathrm{CG} \mathrm{CO}_{2}$ model using the FSS methodology described in section III'C, and the resulting values are compared with experimental data in Figure 4. It is clear from Figure 4 that the use of the CG model leads to a reasonable description of the experimental values with a small overestimate of the interfacial tension corresponding to a shift in the curve by an almost constant amount $(\sim 7 \mathrm{~K})$ over the entire range of temperatures. This constant deviation is related to the overprediction of the critical point with our CG model. A further refinement of the SAFT- $\gamma$ CG Mie model can be made to describe accurately the saturation curve and the interfacial

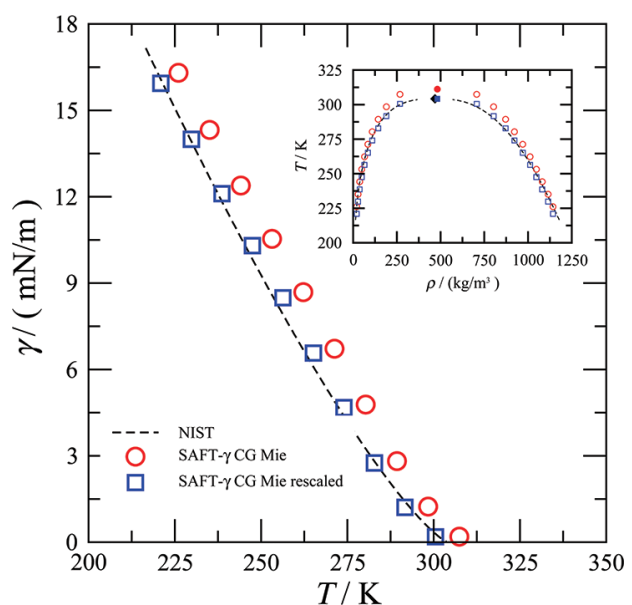

Figure 4. Surface tension as a function of the temperature for $\mathrm{CO}_{2}$. The dashed curve denotes smoothed experimental data from NIST, ${ }^{142,143}$ the circles are the Monte Carlo simulation results for our SAFT- $\gamma$ CG Mie model of $\mathrm{CO}_{2}$, and the squares represent the results of the SAFT- $\gamma$ CG Mie model rescaled to match the critical temperature. The vapor-liquid coexistence curves obtained using the original and rescaled models are shown in the inset.

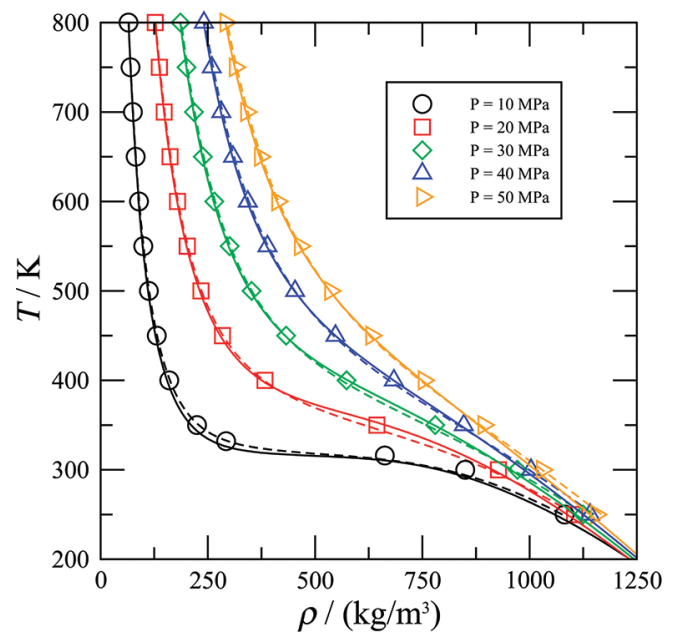

Figure 5. Temperature and pressure dependence of the density of $\mathrm{CO}_{2}$ for supercritical isobars corresponding $P=10,20,30,40$, and $50 \mathrm{MPa}$. The dashed curves denote smoothed experimental data from NIST, ${ }^{142,143}$ the continuous curves the SAFT-VR Mie EoS, and the symbols are the Monte Carlo simulation results for our SAFT- $\gamma$ CG Mie model of $\mathrm{CO}_{2}$.

tension of $\mathrm{CO}_{2}$ simply by ensuring that the model reproduces the experimental critical temperature. One can achieve this with a direct rescaling of the potential energy parameter from $\varepsilon / k_{\mathrm{B}}=$ $361.69 \mathrm{~K}$ to $\varepsilon / k_{\mathrm{B}}=353.55 \mathrm{~K}$, which essentially corresponds to a Guggenheim ${ }^{169}$ corresponding states treatment for the tension, $\gamma=\gamma_{0}\left(1-T / T_{c}\right)^{\mu_{c}}$, where $\gamma_{0}$ is a substance specific "zero temperature" coefficient and $\mu_{\mathrm{c}}=1.26$ is a universal constant. The interfacial tension obtained with the rescaled model is also shown in Figure 4, where an excellent agreement between simulation and experiment is now observed. The saturation densities obtained using the rescaled and unscaled models are depicted in the inset of Figure 4, where a good agreement is seen, apart 


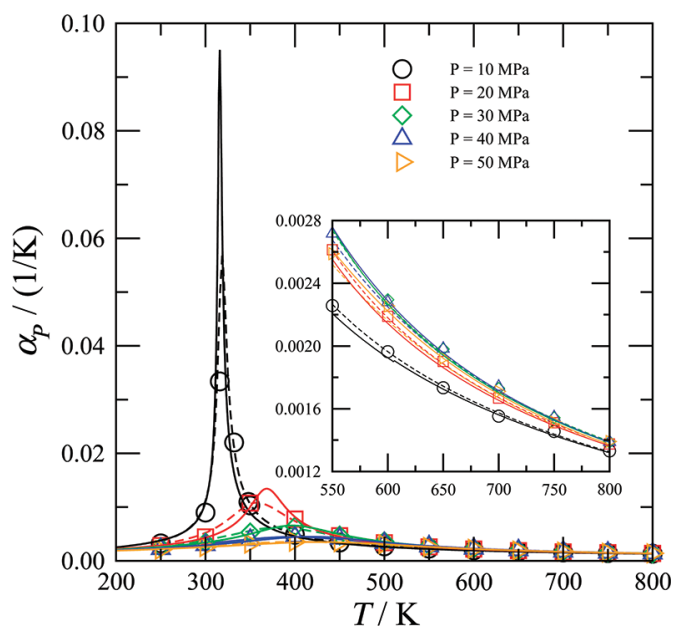

Figure 6. Temperature and pressure dependence of the coefficient of thermal expansion $\alpha_{P}$ for $\mathrm{CO}_{2}$. Legend as in Figure 5.

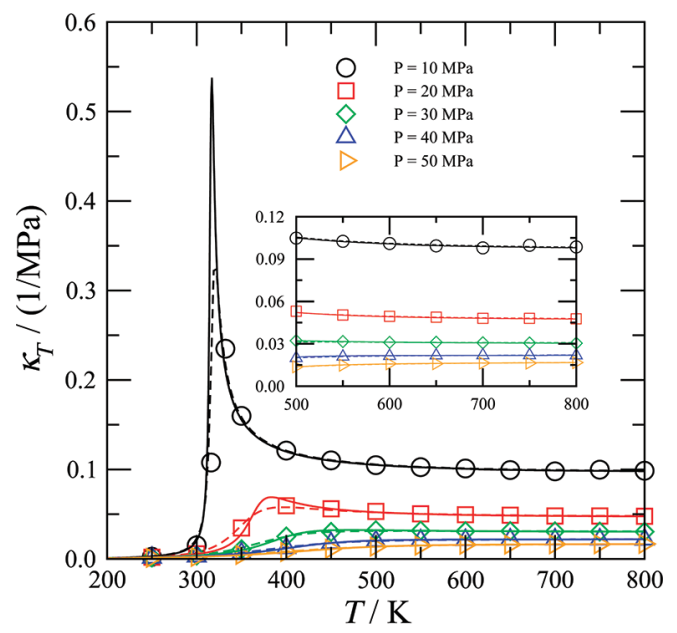

Figure 7. Temperature and pressure dependence of the isothermal compressibility $\kappa_{T}$ for $\mathrm{CO}_{2}$. Legend as in Figure 5 .

from the slight deterioration of the description expected at low temperatures for parameters that have been rescaled to the critical point. In the case of studies of the interfacial properties of $\mathrm{CO}_{2}$ and its mixtures we recommend the use of the rescaled energy parameter.

Lafitte et al. ${ }^{33}$ have shown how by using a Mie potential with variable repulsive and attractive range one is able to represent accurately not only the fluid-phase behavior of a variety of systems but also the single-phase volumetric and second-derivative thermodynamic properties that are of importance in many practical applications. Using NPT-MC simulations, we have calculated the second-derivative properties of $\mathrm{CO}_{2}$ for five supercritical isobars $P=10,20,30,40$, and $50 \mathrm{MPa}$. The results are summarized in Figures 5-10. In Figure 5 we present results for the density as a function of temperature for the five aforementioned isobars. As can be observed, the molecular simulation data obtained with our SAFT- $\gamma$ CG Mie model of $\mathrm{CO}_{2}$ are in good agreement with the experimental values over a broad range of densities, with an $\mathrm{AAD} \%$ for the density of $1.29 \%$. The largest deviation is in the vicinity of the critical region, as one would expect. In the same figure, we make a comparison with the description obtained with the

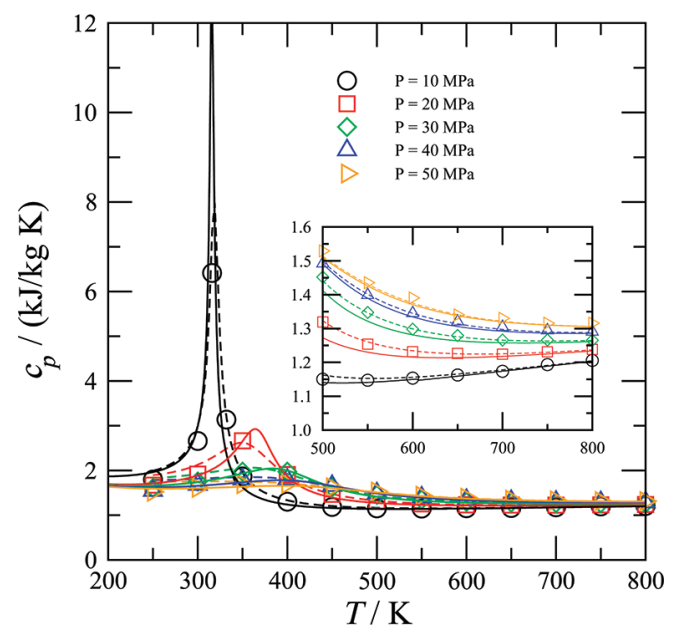

Figure 8. Temperature and pressure dependence of the isobaric heat capacity $C_{P}$ for $\mathrm{CO}_{2}$. Legend as in Figure 5 .

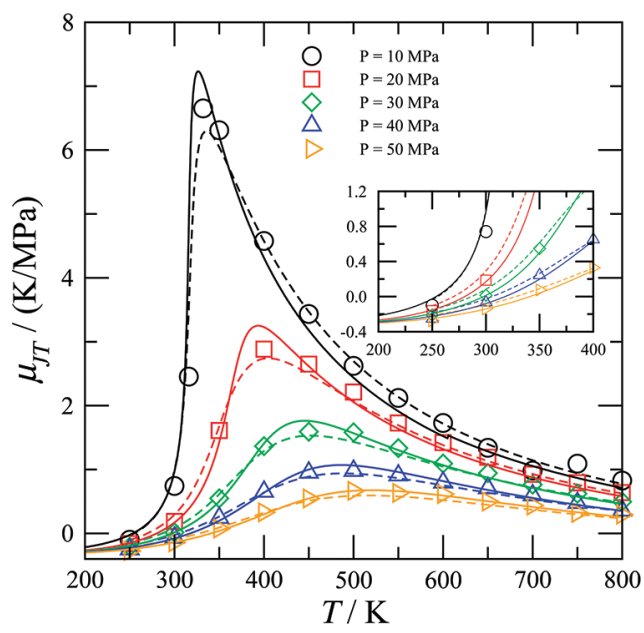

Figure 9. Temperature and pressure dependence of the JouleThomson coefficient $\mu_{\mathrm{JT}}$ for $\mathrm{CO}_{2}$. Legend as in Figure 5 .

SAFT-VR Mie EoS, where it can be observed that the theory provides a good representation of both the experimental and simulation data. Our simulation results are also in good agreement with the values obtained with more sophisticated models: for example, Colina et al. ${ }^{95}$ have reported data for the volumetric properties and second-derivative properties using molecular simulation with the 2CLJQ model. Our data are in close agreement with that of Colina et al., suggesting that (for these properties at least) the electrostatic complexity and nonspherical shape of $\mathrm{CO}_{2}$ can be effectively integrated out and described in an effective sphericalised manner using the adjustable attractive and repulsive exponents of the Mie potential.

The coefficient of thermal expansion, $\alpha_{P}$, and the isothermal compressibility, $\kappa_{T}$, are shown in Figures 6 and 7, respectively. In general, good agreement between the simulation data for the SAFT $-\gamma$ CG Mie model and the experimental values is obtained, with an $\mathrm{AAD} \%$ of $3.90 \%$ and $5.59 \%$ for $\alpha_{P}$ and $\kappa_{T}$, respectively. It is very encouraging to see that one is able to predict accurately the high peaks observed at low pressures and low temperatures (in the vicinity of the critical point) with our CG model. As in the case of the density, the highest $\mathrm{AAD} \%$ s for both properties are 


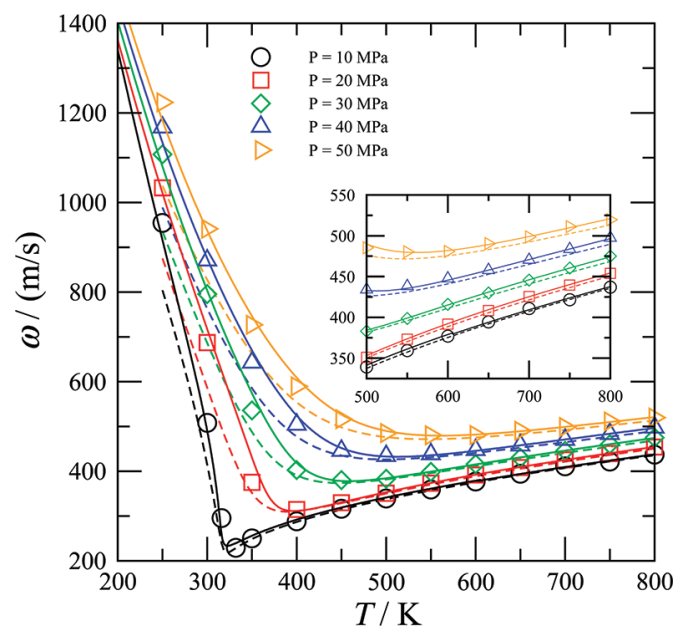

Figure 10. Temperature and pressure dependence of the speed of sound $\omega$ for $\mathrm{CO}_{2}$. Legend as in Figure 5 .

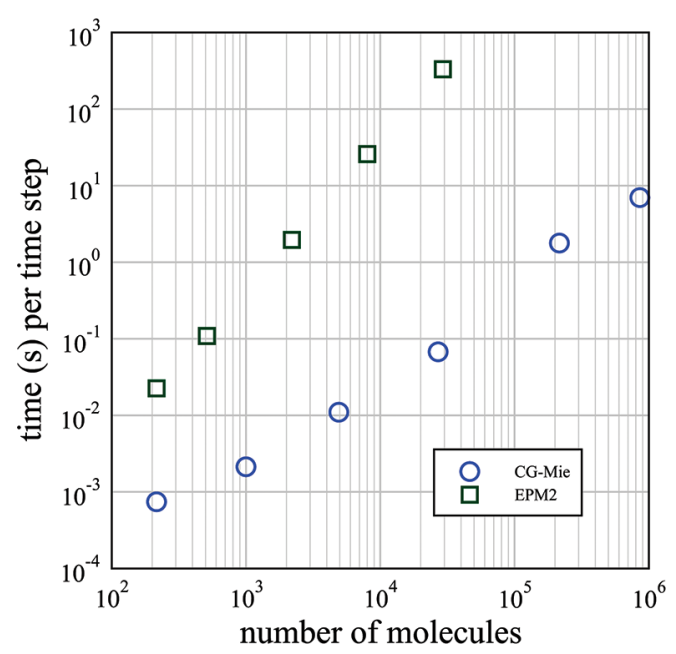

Figure 11. Comparison of the CPU time per time step as a function of the number of molecules using our single-site SAFT- $\gamma$ CG Mie model for $\mathrm{CO}_{2}$ (this work) and the EPM2 model of Harris and Yung. ${ }^{103}$ Canonical ensemble molecular dynamics simulations (NVT) are performed on an $8 \mathrm{CPU} 3 \mathrm{GHz}$ processor desktop computer using DL_POLY v2.18 ${ }^{174}$ compiled with gFORTRAN. The state point is at $T=350 \mathrm{~K}$ and $\rho=336 \mathrm{~kg} / \mathrm{m}^{3}$.

found close to the critical region, where deviations of about $30 \%$ and $20 \%$ can be observed for $\alpha_{P}$ and $\kappa_{T}$, respectively.

In Figure 8 we present the results for the temperature and pressure dependence of the isobaric heat capacity, $C_{p}$, where an $\mathrm{AAD} \%$ of $2.71 \%$ is found between the values obtained with our CG model and experiment. The highest deviations are again seen close to the critical point, decreasing rapidly away from that region. The deviations characterizing our model are comparable to those reported by Colina et al., ${ }^{95}$ who also found an $\mathrm{AAD} \%$ of less than 3\% with the 2CLJQ model.

Finally, in Figures 9 and 10 we present the results for the temperature and pressure dependence of the Joule-Thomson coefficient $\mu_{\mathrm{JT}}$ and speed of sound $\omega$, respectively. As before, very good agreement is found for both properties apart from near the critical region. The description of the speed of sound data obtained with our CG model is commensurate with the results reported by Colina et al. ${ }^{95}$ using the 2CLJQ model, where good agreement with experimental data is found in general. With our model, the overall $\mathrm{AAD} \%$ for the speed of sound is less than $5 \%$. A close inspection of the results for $\mu_{\mathrm{JT}}$ at low temperatures in Figure 8 reveals a good agreement of the values of both the SAFT-VR Mie theory and simulation data for our model compared with experimental data. This region is extremely important for the representation of the Joule-Thomson inversion curve.

The fact that the complex intermolecular potential of $\mathrm{CO}_{2}$ can be represented as a one-site model leads to considerable savings in computational time. For example, for the EPM and EPM2 models developed by Harris and Yung, ${ }^{103}$ the $\mathrm{CO}_{2}$ molecule is modeled as three fused LJ sites with three partial charges embedded in each of the sites. A comparison of the computational performance for molecular dynamics simulations of the fluid with our single-site CG model and the EPM2 model, at one thermodynamic state, is made in Figure 11. The resulting benefits are striking, exemplifying how one can explore longer times and larger length scales using such a CG model. The findings presented in Figure 11 can be rationalized in two ways: for a fixed CPU time per time step one can simulate a system-size that is about 2 orders of magnitude larger with the CG model than with the EPM model; conversely, for a fixed system size one can access simulation times which are two (and in some cases three) orders of magnitude larger with the single-site CG model.

\section{CONCLUSIONS}

In this work we have introduced our new SAFT- $\gamma$ methodology for coarse graining intermolecular potentials. The key feature that sets it apart from common approaches is that we employ a top-down procedure, in which an accurate equation of state (in this case SAFT-VR $\mathrm{Mie}^{34}$ ) is used to estimate the molecular parameters from experimental macroscopic fluid-phase properties. The robustness of our approach lies in the fact that the effective intermolecular potentials developed in this way are not state dependent, which is an issue commonly faced with bottomup CG approaches.

The version of SAFT-VR Mie used in our work has thus been shown to be powerful tool not only in the description of the fluidphase behavior of different systems but also as a global framework for the representation of complex intermolecular potential functions with a much simpler Mie form. The approach still involves the estimation of the potential parameters using some macroscopic experimental input. However, this is an efficient procedure as the theory is algebraic and a large amount of data can be included in estimating the parameters for a broad range of thermodynamic states, involving only a few minutes (or even seconds) of CPU time. Using this approach, it has been possible to obtain optimized molecular parameters that are not statedependent. This is a step change in comparison with the common bottom-up approaches, in which computationally expensive simulations of high-resolution models are required to establish the CG models.

We have outlined the methodology by proposing a single-site spherical CG model for the $\mathrm{CO}_{2}$ molecule. This molecule is interesting not only from the technological and environmental point of view but also as a nontrivial test case as its potential function is sufficiently complex, involving nonuniform dispersion force centers, electrostatic terms, and a nonspherical shape. The main aim of the current paper is to show how $\mathrm{CO}_{2}$ can be described, using the SAFT-VR Mie equation of state with a single-site 
SAFT- $\gamma$ CG Mie force-field with appropriate choices of the attraction and the repulsion parameters. The simulation and theoretical results for the CG model are in good agreement with most of the experimental data, apart from the vicinity of the critical region. The description obtained with our CG model also compares favorably with that for more sophisticated models of $\mathrm{CO}_{2}$. One remarkable feature is the improved prediction of the vapor pressure with our model. This is one of the most elusive properties to model with CG models, but one that is important from an engineering perspective. Even the de facto model for $\mathrm{CO}_{2}$, the EPM2 model, fails to provide a good description of the vapor pressure. The adequacy of our $\mathrm{CG}$ model for $\mathrm{CO}_{2}$ has also been assessed in its description of the volumetric and secondderivative properties. We have simulated different supercritical isobars, finding good overall agreement with experiments, and with the corresponding representation obtained with the more complex models, ${ }^{170}$ though some deviation in the critical region is observed.

The present methodology is not limited with respect to the type of molecule that is considered and may be applied in a straightforward fashion to large macromolecules and to mixtures. In the forthcoming work we will demonstrate how long chain molecules such as $n$-alkanes can be described as CG chains formed from Mie segments. The methodology can also be extended to treat molecules formed from heteronuclear Mie sites of different type by using the SAFT- $\gamma$ equation of state, which is an extension of the homonuclear SAFT-VR approach, suitable for molecules comprising chemically distinct groups (e.g., see refs 35 and 36 for a description of the group contribution theory for molecules formed from different square-well segments). The algebraic SAFT- $\gamma$ equation of state thus enables the development of force-fields for both coarse-grained and united-atom representations of complex molecules. We are currently expanding the SAFT- $\gamma$ force field (based on Mie segments) for a wide range of compounds of varying chemical nature.

\section{APPENDIX}

In this Appendix, we summarize the key relations of the SAFTVR Mie equation of state that is used in this work to develop the CG SAFT- $\gamma$ force field for $\mathrm{CO}_{2}$. For a complete derivation of the theory the reader is directed to our forthcoming paper. ${ }^{34}$

The general formulation of the (dimensionless) Helmholtz free energy for a nonassociating chain fluid is expressed in the usual SAFT manner as

$$
a=a^{\mathrm{IDEAL}}+a^{\mathrm{MONO}}+a^{\mathrm{CHAIN}}
$$

where $a=A \beta / N, A$ being the total Helmholtz free energy, and $N$ is the total number of molecules. In this equation $a^{\mathrm{IDEAL}}$ is the contribution of the ideal free energy, $a^{\mathrm{MONO}}$ is the residual free energy due to the monomer segments, and $a^{\mathrm{CHAIN}}$ is the contribution due to the formation of the chains of monomers.

A1. Ideal Contribution. The ideal gas contribution is given in the usual form as

$$
a^{\text {IDEAL }}=\ln \left(\rho \Lambda^{3}\right)-1
$$

where $\rho$ is the number density of chain molecules and $\Lambda$ is a de Broglie wavelength that incorporates all of the translational, rotational, and vibrational kinetic contributions of the molecules.
A2. Monomer Contribution. The monomer contribution for a chain composed of $m_{\mathrm{s}}$ segments is

$$
a^{\mathrm{MONO}}=m_{\mathrm{s}} a^{\mathrm{M}}
$$

where $a^{\mathrm{M}}=A^{\mathrm{M}} \beta / N_{\mathrm{s}}$ is the residual Helmholtz free energy per monomer and $N_{s}$ is the number of spherical segments. This term is expressed as a series expansion in the inverse of the temperature $\beta$ up to third-order: ${ }^{171}$

$$
a^{\mathrm{M}}=a^{\mathrm{HS}}+\beta a_{1}+\beta^{2} a_{2}+\beta^{3} a_{3}
$$

In this relation $a^{\mathrm{HS}}$ is the Helmholtz free energy of a hardsphere (HS) fluid of diameter $d$, which is obtained from the Carnahan and Starling relation as ${ }^{172}$

$$
a^{\mathrm{HS}}=\frac{4 \eta-3 \eta^{2}}{(1-\eta)^{2}}
$$

where $\eta=(\pi / 6) \rho_{\mathrm{s}} d^{3} ; \rho_{\mathrm{s}}$ is the number density of spherical segments. According to the Barker and Henderson theory,,$^{31,34,171}$ the effective diameter $d$ can be obtained as

$$
d=\int_{0}^{\sigma}\left[1-\exp \left(-\beta u^{\mathrm{Mie}}(r)\right)\right] \mathrm{d} r
$$

The first perturbation term $a_{1}$ can be obtained with the following compact expression:

$$
\begin{aligned}
a_{1}= & \mathcal{S}\left[x_{0}^{\lambda_{\mathrm{a}}}\left\{a_{1}^{\mathrm{s}}\left(\eta ; \lambda_{\mathrm{a}}\right)+B\left(\eta ; \lambda_{\mathrm{a}}\right)\right\}-x_{0}^{\lambda_{\mathrm{r}}}\left\{a_{1}^{\mathrm{s}}\left(\eta ; \lambda_{\mathrm{r}}\right)\right.\right. \\
& \left.\left.+B\left(\eta ; \lambda_{\mathrm{r}}\right)\right\}\right]
\end{aligned}
$$

where $x_{0}=\sigma / d, \mathcal{O}$ is the Mie potential coefficient defined in eq 3 , and

$$
B(\eta ; \lambda)=12 \eta \varepsilon\left(\frac{1-\eta / 2}{(1-\eta)^{3}} I_{\lambda}(\lambda)-\frac{9 \eta(1+\eta)}{2(1-\eta)^{3}} J_{\lambda}(\lambda)\right)
$$

Note that to calculate the first- and second-order terms, as given by eqs 33 and 40 , one needs to obtain an analytical expression for the mean-attractive energy $a_{1}^{s}(\lambda)$ of a Sutherland potential of variable range. These expressions are obtained using a SAFT-VR ${ }^{31,32}$ treatment, which gives the following compact expression:

$$
a_{1}^{\mathrm{s}}(\lambda)=-12 \varepsilon \eta\left(\frac{1}{\lambda-3}\right) \frac{1-\eta_{\mathrm{eff}} / 2}{\left(1-\eta_{\mathrm{eff}}\right)^{3}}
$$

where the effective packing fraction $\eta_{\text {eff }}$ is parameterized for the range $5<\lambda \leq 100$. The mean-attractive energy $a_{1}^{\text {s }}(\lambda)$ calculated in this way is as accurate as computer simulation results, ${ }^{34}$ with the effective density parameterized as

$$
\eta_{\text {eff }}=c_{1} \eta+c_{2} \eta^{2}+c_{3} \eta^{3}+c_{4} \eta^{4}
$$

where

$$
\left(\begin{array}{l}
c_{1} \\
c_{2} \\
c_{3} \\
c_{4}
\end{array}\right)=\left(\begin{array}{cccc}
0.81096 & 1.7888 & -37.578 & 92.284 \\
1.0205 & -19.341 & 151.26 & -463.50 \\
-1.9057 & 22.845 & -228.14 & 973.92 \\
1.0885 & -6.1962 & 106.98 & -677.64
\end{array}\right)\left(\begin{array}{c}
1 \\
1 / \lambda \\
1 / \lambda^{2} \\
1 / \lambda^{3}
\end{array}\right)
$$

In eq 34 the functions $I_{\lambda}$ and $J_{\lambda}$ are two integrals that depend on the molecular parameters of the Mie potential and are given 
by

$$
I_{\lambda}(\lambda)=\int_{1}^{x_{0}} \frac{x^{2}}{x^{\lambda}} \mathrm{d} x=-\frac{\left(x_{0}\right)^{-\lambda+3}-1}{\lambda-3}
$$

and

$$
\begin{aligned}
J_{\lambda}(\lambda) & =\int_{1}^{x_{0}} \frac{\left(x^{3}-1\right)}{x^{\lambda}} \mathrm{d} x \\
& =-\frac{\left(x_{0}\right)^{-\lambda+4}(\lambda-4)-\left(x_{0}\right)^{-\lambda+3}(\lambda-3)-1}{(\lambda-3)(\lambda-4)}
\end{aligned}
$$

The second perturbation term $a_{2}$ is evaluated with a modified macroscopic compressibility approximation (MCA), ${ }^{34}$ which can be written as a function of the mean-attractive energies of hard-core Sutherland potentials $a_{1}^{s}$ as

$$
\begin{aligned}
a_{2}= & \frac{1}{2} K^{\mathrm{HS}}(1+\chi) \varepsilon \mathcal{C}^{2}\left[x _ { 0 } ^ { 2 \lambda _ { \mathrm { a } } } \left\{a_{1}^{\mathrm{s}}\left(\eta ; 2 \lambda_{\mathrm{a}}\right)\right.\right. \\
& \left.+B\left(\eta ; 2 \lambda_{\mathrm{a}}\right)\right\}-2 x_{0}^{\left(\lambda_{\mathrm{a}}+\lambda_{\mathrm{r}}\right)}\left\{a_{1}^{\mathrm{s}}\left(\eta ; \lambda_{\mathrm{a}}+\lambda_{\mathrm{r}}\right)\right. \\
& \left.\left.+B\left(\eta ; \lambda_{\mathrm{a}}+\lambda_{\mathrm{r}}\right)\right\}+x_{0}^{2 \lambda_{\mathrm{r}}}\left\{a_{1}^{\mathrm{s}}\left(\eta ; 2 \lambda_{\mathrm{r}}\right)+B\left(\eta ; \lambda_{\mathrm{r}}\right)\right\}\right)
\end{aligned}
$$

where $K^{\mathrm{HS}}$ is the Carnahan and Starling ${ }^{172}$ expression for the isothermal compressibility given by

$$
K^{\mathrm{HS}}=\frac{(1-\eta)^{4}}{1+4 \eta+4 \eta^{2}-4 \eta^{3}+\eta^{4}}
$$

and $\chi$ is an empirical function of $\eta$ introduced to reproduce the fluctuation term of the Mie potential obtained by Monte Carlo simulation. ${ }^{34}$ This correction is expressed as

$$
\chi=f_{1}(\alpha) \eta+f_{2}(\alpha) \eta^{5}+f_{3}(\alpha) \eta^{8}
$$

where $\alpha$ represents the reduced van der Waals attractive constant of the Mie potential,

$$
\alpha=\mathcal{C}\left(\frac{1}{\lambda_{\mathrm{a}}-3}-\frac{1}{\lambda_{\mathrm{r}}-3}\right)
$$

The third-order term in the Helmholtz free energy expansion is given by the following empirical function:

$$
a_{3}=\varepsilon^{3} f_{4}(\alpha) \eta x_{0}{ }^{3} \exp \left(f_{5}(\alpha) \eta x_{0}{ }^{3}+f_{6}(\alpha) \eta^{2} x_{0}{ }^{6}\right)
$$

which depends on the Mie exponents $\lambda_{\mathrm{a}}$ and $\lambda_{\mathrm{r}}$ through the constant $\alpha$. The functions $f_{i}$ are defined as follows:

$f_{i}(\alpha)=\sum_{n=0}^{n=3} \phi_{i, n} \alpha^{n} /\left(1+\sum_{n=4}^{n=6} \phi_{i, n} \alpha^{n-3}\right) \quad$ for $\quad i=1, \ldots, 6$

The values of the coefficients $\phi_{i, n}$ are reported in Table 3 .

A3. Chain Contribution. Assuming that the segments are bonded together at $r=\sigma$, i.e., that the model consists of freely jointed chains of tangent segments, the residual contribution to the Helmholtz free energy is given by

$$
a^{\text {CHAIN }}=-\left(m_{s}-1\right) \ln g^{\mathrm{Mie}}(\sigma)
$$

where $g^{\mathrm{Mie}}$ is the radial distribution function (RDF) at contact of the monomer Mie fluid, which is given by the following expression: ${ }^{34}$

$$
\begin{aligned}
g^{\mathrm{Mie}}(\sigma)= & g_{\mathrm{d}}^{\mathrm{HS}}(\sigma) \exp \left(\beta \varepsilon g_{1}(\sigma) / g_{\mathrm{d}}^{\mathrm{HS}}(\sigma)\right. \\
& \left.+(\beta \varepsilon)^{2} g_{2}(\sigma) / g_{\mathrm{d}}^{\mathrm{HS}}(\sigma)\right)
\end{aligned}
$$

where $g_{\mathrm{d}}^{\mathrm{HS}}(\sigma)$ refers to the RDF of a fluid of HS of diameter $d$ evaluated at diameter $\sigma$. This is obtained using the expression of Boublik, ${ }^{173}$

$$
g_{\mathrm{d}}^{\mathrm{HS}}\left(x_{0}\right)=\exp \left(k_{0}+k_{1} x_{0}+k_{2} x_{0}{ }^{2}+k_{3}{ }^{3}\right)
$$

In this expression, the density-dependent coefficients $k_{i}$ are given by

$$
\begin{aligned}
& k_{0}=-\ln (1-\eta)+\frac{42 \eta-}{2} \\
& k_{1}=\frac{\left(\eta^{4}+6 \eta^{2}-12 \eta\right)}{2(1-\eta)^{3}} \\
& k_{2}=\frac{-3 \eta^{2}}{8(1-\eta)^{2}} \\
& k_{3}=\frac{\left(-\eta^{4}+3 \eta^{2}+3 \eta\right)}{6(1-\eta)^{3}}
\end{aligned}
$$

The first-order radial distribution function at contact in eq 47 is expressed in terms of the first-order perturbation terms of the Helmholtz free energy of Sutherland $\left(a_{1}^{s}\right)$ and Mie $\left(a_{1}\right)$ potentials as follows:

$$
\begin{aligned}
g_{1}(\sigma)= & \frac{1}{2 \pi \varepsilon d^{3}}\left[3 \frac{\partial a_{1}}{\partial \rho_{\mathrm{s}}}-\mathcal{C} \lambda_{\mathrm{a}} x_{0}^{\lambda_{\mathrm{a}}} \frac{a_{1}^{\mathrm{s}}\left(\eta ; \lambda_{\mathrm{a}}\right)+B\left(\eta ; \lambda_{\mathrm{a}}\right)}{\rho_{\mathrm{s}}}\right. \\
& \left.+C \lambda_{\mathrm{r}} x_{0}^{\lambda_{\mathrm{r}}} \frac{a_{1}^{\mathrm{s}}\left(\eta ; \lambda_{\mathrm{r}}\right)+B\left(\eta ; \lambda_{\mathrm{r}}\right)}{\rho_{\mathrm{s}}}\right]
\end{aligned}
$$

The second-order term in the radial distribution function expansion is based on a corrected MCA approximation,

$$
g_{2}(\sigma)=\left(1+\gamma_{\mathrm{c}}\right) g_{2}^{\mathrm{MCA}}(\sigma)
$$

where

$$
\begin{aligned}
g_{2}^{\mathrm{MCA}}(\sigma)= & \frac{1}{2 \pi \varepsilon^{2} d^{3}}\left[\frac{3}{1+\chi} \frac{\partial a_{2}}{\partial \rho_{\mathrm{s}}}\right. \\
& -\varepsilon K^{\mathrm{HS}} \mathcal{O}^{2} \lambda_{\mathrm{r}} x_{0}^{2 \lambda_{\mathrm{r}}} \frac{a_{1}^{\mathrm{s}}\left(\eta ; 2 \lambda_{\mathrm{r}}\right)+B\left(\eta ; 2 \lambda_{\mathrm{r}}\right)}{\rho_{\mathrm{s}}} \\
& +\varepsilon K^{\mathrm{HS}} \mathcal{O}^{2}\left(\lambda_{\mathrm{r}}+\lambda_{\mathrm{a}}\right) x_{0}^{\lambda_{\mathrm{r}}+\lambda_{\mathrm{a}}} \\
& \times \frac{a_{1}^{\mathrm{s}}\left(\eta ; \lambda_{\mathrm{r}}+\lambda_{\mathrm{a}}\right)+B\left(\eta ; \lambda_{\mathrm{r}}+\lambda_{\mathrm{a}}\right)}{\rho_{\mathrm{s}}} \\
& \left.-\varepsilon K^{\mathrm{HS}} \mathcal{O}^{2} \lambda_{\mathrm{a}} x_{0}^{2 \lambda_{\mathrm{a}}} \frac{a_{1}^{\mathrm{s}}\left(\eta ; 2 \lambda_{\mathrm{a}}\right)+B\left(\eta ; 2 \lambda_{\mathrm{a}}\right)}{\rho_{\mathrm{s}}}\right]
\end{aligned}
$$

The empirical correction $\gamma_{c}$ is chosen to capture the positive slope of the radial distribution function at contact for long-range 
Table 3. Coefficients $\phi_{i, n}$ for eqs 42 and 56

\begin{tabular}{|c|c|c|c|c|c|c|c|}
\hline$n$ & $\phi_{1, n}$ & $\phi_{2, n}$ & $\phi_{3, n}$ & $\phi_{4, n}$ & $\phi_{5, n}$ & $\phi_{6, n}$ & $\phi_{7, n}$ \\
\hline 0 & 7.5365557 & -359.44 & 1550.9 & -1.19932 & -1911.28 & 9236.9 & 10 \\
\hline 1 & -37.60463 & 1825.6 & -5070.1 & 9.063632 & 2139.175 & -129430 & 10 \\
\hline 2 & 71.745953 & -3168.0 & 6534.6 & -17.9482 & -51320.7 & 357230 & 0.57 \\
\hline 3 & -46.83552 & 1884.2 & -3288.7 & 11.34027 & 37064.54 & -315530 & -6.7 \\
\hline 4 & -2.467982 & -0.82376 & -2.7171 & 20.52142 & 1103.742 & 1390.2 & -8 \\
\hline 5 & -0.50272 & -3.1935 & 2.0883 & -56.6377 & -3264.61 & -4518.2 & \\
\hline 6 & 8.0956883 & 3.7090 & 0 & 40.53683 & 2556.181 & 4241.6 & \\
\hline
\end{tabular}

Mie potentials at low temperature and low density. It is a function of both density and temperature as well as the Mie exponents $\left(\lambda_{\mathrm{a}}, \lambda_{\mathrm{r}}\right)$ through

$$
\begin{aligned}
\gamma_{c}= & \phi_{7,0}\left(-\tanh \left(\phi_{7,1}\left(\phi_{7,2}-\alpha\right)\right)\right. \\
& +1) \eta \theta \exp \left(\phi_{7,3} \eta+\phi_{7,4} \eta^{2}\right)
\end{aligned}
$$

where $\theta=\exp (\beta \varepsilon)-1, \alpha$ is given by eq 43 , and the coefficients $\phi_{7, i}$ are given in Table 3 .

\section{AUTHOR INFORMATION}

\section{Corresponding Author}

*E-mail: e.muller@imperial.ac.uk.

\section{ACKNOWLEDGMENT}

We thank Dr. Andres Mejía (Universidad de Concepción, Chile) for stimulating discussions. C.A. and T.L. are very grateful to the Engineering and Physical Sciences Research Council (EPSRC) of the U.K. for the award of postdoctoral fellowships. Additional funding to the Molecular Systems Engineering Group from the EPSRC (grants GR/T17595, GR/N35991, and EP/ E016340), the Joint Research Equipment Initiative (JREI) (GR/ M94426), and the Royal Society-Wolfson Foundation refurbishment scheme is also gratefully acknowledged. Simulations were performed using the facilities of the Imperial College High Performance Computing Service.

\section{REFERENCES}

(1) Jorgensen, W. L.; Maxwell, D. S.; Tirado-Rives, J. J. Am. Chem. Soc. 1996, 118, 11225 .

(2) Martin, M. G.; Siepmann, J. I. J. Phys. Chem. B 1998, 102, 2569.

(3) Search done on ISI Web of Science, on 20.07.10 searching articles from 2009. Search parameters "molecular dynamics or Monte Carlo or Molecular Dynamics" gave 20265 hits in 1442223 records.

(4) Moore, G. E. Electronics 1965, 38 (8), 114.

(5) Kadau, K.; Germann, T. C.; Lomdahl, P. S. Int. J. Mod. Phys. C 2006, 17, 1755 .

(6) Germann, T. C.; Kadau, K. Int. J. Mod. Phys. C 2008, 19, 1315.

(7) Voth, G. Coarse-Graining of Condensed Phase and Biomolecular Systems; CRC Press: Boca Raton, FL, 2009.

(8) Faller, R. Phys. Chem. Chem. Phys. 2009, 11, 1867.

(9) Peter, C.; Kremer, K. Faraday Discuss. 2010, 144, 9.

(10) Klein, M. L.; Shinoda, W. Science 2008, 321, 798.

(11) McCullagh, M.; Prytkova, T.; Tonzani, S.; Winter, N. D.; Schatz, G. C. J. Phys. Chem. B 2008, 112, 10388.

(12) Noid, W. G.; Chu, J.-W.; Ayton, G. S.; Krishna, V.; Izvekov, S.; Voth, G. A.; Das, A.; Andersen, H. C. J. Chem. Phys. 2008, 128, 244114.

(13) Izvekov, S.; Parrinello, M.; Burnham, C. J.; Voth, G. A. J. Chem. Phys. 2004, 120, 10896.
(14) Izvekov, S.; Voth, G. A. J. Phys. Chem. B 2005, 109, 2469.

(15) Shelley, J. C.; Shelley, M. Y.; Reeder, R. C.; Bandyopadhyay, S.; Klein, M. L. J. Phys. Chem. B 2001, 105, 4464.

(16) Reith, D.; Putz, M.; Müller-Plathe, F. J. Comput. Chem. 2003, 24, 1624.

(17) Nielsen, S. O.; Lopez, C. F.; Srinivas, G.; Klein, M. L. J. Chem. Phys. 2003, 119, 7043.

(18) Marrink, S. J.; Risselada, H. J.; Yefimov, S.; Tieleman, D. P.; de Vries, A. H. J. Phys. Chem. B 2007, 111, 7812.

(19) Müller, E. A.; Gelb, L. D. Ind. Eng. Chem. Res. 2003, 42, 4123.

(20) Albo, S.; Müller, E. A. J. Phys. Chem. B 2003, 107, 1672.

(21) Virnau, P.; Müller, M.; MacDowell, L. G.; Binder, K. J. Chem. Phys. 2004, 121, 2169.

(22) Mognetti, B. M.; Yelash, L.; Virnau, P.; Paul, W.; Binder, K.; Müller, M.; MacDowell, L. G. J. Chem. Phys. 2008, 128, 104501.

(23) Mognetti, B. M.; Oettel, M.; Yelash, L.; Virnau, P.; Paul, W.; Binder, K. Phys. Rev. E 2008, 77, 041506.

(24) Mognetti, B. M.; Virnau, P.; Yelash, L.; Paul, W.; Binder, K.; Müller, M.; MacDowell, L. G. J. Chem. Phys. 2009, 130, 044101.

(25) Mognetti, B. M.; Virnau, P.; Yelash, L.; Paul, W.; Binder, K.; Müller, M.; MacDowell, L. G. Phys. Chem. Chem. Phys. 2009, 11, 1923.

(26) Shell, M. S. J. Chem. Phys. 2008, 129, 144108.

(27) Shinoda, W.; Devane, R.; Klein, M. L. Mol. Simul. 2007, 33, 27.

(28) DeVane, R.; Shinoda, W.; Moore, P. B.; Klein, M. L. J. Chem. Theory Comput. 2009, 5, 2115.

(29) Chiu, S.-W.; Scott, H. L.; Jakobsson, E. J. Chem. Theory Comput. 2010, 6, 851 .

(30) Maerzke, K. A.; Siepmann, J. I. J. Phys. Chem. B 2011, 115, 3452.

(31) Gil-Villegas, A.; Galindo, A.; Whitehead, P. J.; Mills, S. J.; Jackson, G.; Burgess, A. N. J. Chem. Phys. 1997, 106, 4168.

(32) Galindo, A.; Davies, L. A.; Gil-Villegas, A.; Jackson, G. Mol. Phys. 1998, 93, 241.

(33) Lafitte, T.; Bessieres, B.; Piñeiro, M. M.; Daridon, J.-L. J. Chem. Phys. 2006, 124, 024509.

(34) Lafitte, T.; Apostolakou, A.; Avendaño, C.; Galindo, A.; Adjiman, C. S.; Müller, E. A.; Jackson, G. Manuscript in preparation, 2011.

(35) Lymperiadis, A.; Adjiman, C. S.; Galindo, A.; Jackson, G. J. Chem. Phys. 2007, 127, 234903.

(36) Lymperiadis, A.; Adjiman, C. S.; Jackson, G.; Galindo, A. Fluid Phase Equilib. 2008, 274, 85.

(37) Papaioannou, V.; Lafitte, T.; Avendaño, C.; Adjiman, C. S.; Jackson, G.; Müller, E. A.; Galindo, A. Manuscript in preparation, 2011.

(38) Mie, G. Ann. Phys. 1903, 316, 657.

(39) Jones, J. E. Proc. R. Soc. London Ser. A-Math. Phys. Eng. Sci. 1924, 106, 463.

(40) Chapman, W. G.; Gubbins, K. E.; Jackson, G.; Radosz, M. Fluid Phase Equilib. 1989, 52, 31.

(41) Chapman, W. G.; Gubbins, K. E.; Jackson, G.; Radosz, M. Ind. Eng. Chem. Res. 1990, 29, 1709.

(42) Müller, E. A.; Gubbins, K. E. Ind. Eng. Chem. Res. 2001, 40, 2193.

(43) Economou, I. G. Ind. Eng. Chem. Res. 2002, 41, 953.

(44) Tan, S. P.; Adidharma, H.; Radosz, M. Ind. Eng. Chem. Res. 2008, 47, 8063. 
(45) McCabe, C.; Galindo, A. SAFT associating fluids and fluid mixtures. In Applied Thermodynamics of Fluids; Goodwin, A., Sengers, J. V., Peters, C. J., Eds.; Royal Society of Chemistry, London, 2010; Chapter 8.

(46) Müller, E. A.; Gubbins, K. E. Ind. Eng. Chem. Res. 1995, $34,3662$.

(47) Davies, L. A.; Gil-Villegas, A.; Jackson, G. Int. J. Thermophys. 1998, 19, 675 .

(48) Davies, L. A.; Gil-Villegas, A.; Jackson, G. J. Chem. Phys. 1999, 111,8659

(49) Potoff, J. J.; Bernard-Brunel, D. A. J. Phys. Chem. B 2009, 113,14725 .

(50) Okumura, H.; Yonezawa, F. J. Chem. Phys. 2000, 113, 9162.

(51) Gordon, P. A. J. Chem. Phys. 2006, 125, 014504.

(52) Gibbons, T. G.; Klein, M. L. J. Chem. Phys. 1974, 60, 112.

(53) He, X.; Shinoda, W.; DeVane, R.; Klein, M. L. Mol. Phys. 2010, 108, 2007.

(54) Shelley, J. C.; Shelley, M. Y.; Reeder, R. C.; Bandyopadhyay, S.; Moore, P. B.; Klein, M. L. J. Phys. Chem. B 2001, 105, 9785.

(55) Srinivas, G.; Shelley, J. C.; Nielsen, S. O.; Discher, D. E.; Klein, M. L. J. Phys. Chem. B 2004, 108, 8153.

(56) Bhargava, B. L.; Devane, R.; Klein, M. L.; Balasubramanian, S. Soft Matter 2007, 3, 1395.

(57) De Vane, R.; Klein, M. L.; Chiu, C.-c.; Nielsen, S. O.; Shinoda, W.; Moore, P. B. J. Phys. Chem. B 2010, 114, 6386.

(58) Reed, T. M.; Gubbins, K. E. Applied statistical mechanics; McGraw-HillButterworth-Heinemann: Stoneham, 1973.

(59) Reid, R. C.; Prausnitz, J. M.; Poling, B. E. The properties of gases and liquids, 4th ed.; McGraw-Hill: New York, 1987.

(60) Ruckenstein, E.; Liu, H. Q. Ind. Eng. Chem. Res. 1997, 36, 3927.

(61) Liu, H. Q.; Silva, C. M.; Macedo, E. A. Ind. Eng. Chem. Res. 1997, 36, 246.

(62) Yu, Y. X.; Gao, G. H. Fluid Phase Equilib. 1999, 166, 111.

(63) Dariva, C.; Coelho, L. A. F.; Oliveira, J. V. Fluid Phase Equilib. 1999, 158, 1045.

(64) Iwai, Y.; Uchida, H.; Arai, Y.; Mori, Y. Fluid Phase Equilib. 1998, 144, 233.

(65) Zhu, Y.; Lu, X. H.; Zhou, J.; Wang, Y. R.; Shi, J. Fluid Phase Equilib. 2002, 194, 1141.

(66) Iwai, Y.; Koga, Y.; Hata, Y.; Uchida, H.; Arai, Y. Fluid Phase Equilib. 1995, 104, 403.

(67) Ravi, R.; Guruprasad, V. Ind. Eng. Chem. Res. 2008, 47, 1297.

(68) Iwai, Y.; Uchida, H.; Koga, Y.; Arai, Y.; Mori, Y. Ind. Eng. Chem. Res. 1996, 35, 3782.

(69) Guo, M. X.; Lu, B. C. Y. Thermochim. Acta 1997, 297, 187.

(70) Koga, Y.; Iwai, Y.; Yamamoto, M.; Arai, Y. Fluid Phase Equilib. $1997,131,83$.

(71) Nakanishi, K. Fluid Phase Equilib. 1998, 144, 217.

(72) Yamamoto, M.; Iwai, Y.; Arai, Y. Fluid Phase Equilib. 1999, 163, 165.

(73) Iwai, Y.; Mori, Y.; Arai, Y. Fluid Phase Equilib. 2000, 167, 33.

(74) Iwai, Y.; Higashi, H.; Uchida, H.; Arai, Y. Fluid Phase Equilib. 1997, 127, 251.

(75) Higashi, H.; Iwai, Y.; Uchida, H.; Arai, Y. J. Supercrit. Fluids 1998, 13, 93.

(76) Higashi, H.; Iwai, Y.; Arai, Y. Ind. Eng. Chem. Res. 2000, $39,4567$.

(77) Zhou, J.; Lu, X. H.; Wang, Y. R.; Shi, J. Fluid Phase Equilib. 2000, 172, 279.

(78) Nitta, T.; Shigeta, T. Fluid Phase Equilib. 1998, 144, 245.

(79) Kurniawan, Y.; Bhatia, S. K.; Rudolph, V. AIChE J. 2006, $52,957$.

(80) Du, Q.; Yang, Z.; Yang, N.; Yang, X. Ind. Eng. Chem. Res. 2010, 49,8271 .

(81) Buckingham, A. D. Q. Rev. Chem. Soc. 1959, 13, 183.

(82) Vrabec, J.; Fischer, J. AIChE J. 1997, 43, 212.

(83) Nouacer, M.; Shing, K. S. Mol. Simul. 1989, 2, 55.

(84) Johnson, J. D.; Shaw, M. S. J. Chem. Phys. 1985, 83, 1271.
(85) Matthews, G. P.; Townsend, A. Chem. Phys. Lett. 1989, $155,518$.

(86) Möller, D.; Fischer, J. Fluid Phase Equilib. 1994, 100, 35-61.

(87) Liu, A. P.; Beck, T. L. J. Phys. Chem. B 1998, 102, 7627.

(88) Vrabec, J.; Stoll, J.; Hasse, H. J. Phys. Chem. B 2001, 105, 12126.

(89) Agrawal, P. M.; Sorescu, D. C.; Rice, B. M.; Thompson, D. L. Fluid Phase Equilib. 1999, 155, 177.

(90) Agrawal, P. M.; Rice, B. M.; Sorescu, D. C.; Thompson, D. L. Fluid Phase Equilib. 1999, 166, 1.

(91) Agrawal, P. M.; Rice, B. M.; Sorescu, D. C.; Thompson, D. L. Fluid Phase Equilib. 2001, 187, 139.

(92) Heuchel, M.; Davies, G. M.; Buss, E.; Seaton, N. A. Langmuir 1999, 15, 8695 .

(93) Müller, E. A. J. Phys. Chem. B 2008, 112, 8999.

(94) Chacin, A.; Vazquez, J. M.; Müller, E. A. Fluid Phase Equilib. 1999, 165, 147.

(95) Colina, C. M.; Olivera-Fuentes, C. G.; Siperstein, F. R.; Lisal, M.; Gubbins, K. E. Mol. Simul. 2003, 29, 405.

(96) Vrabec, J.; Kedia, G. K.; Hasse, H. Cryogenics 2005, 45, 253.

(97) Vrabec, J.; Huang, Y. L.; Hasse, H. Fluid Phase Equilib. 2009, 279, 120.

(98) Huang, Y. L.; Vrabec, J.; Hasse, H. Fluid Phase Equilib. 2009, 287, 62 .

(99) Garzon, B.; Lago, S.; Vega, C.; De Miguel, E.; Rull, L. F. J. Chem. Phys. 1994, 101, 4166.

(100) Murthy, C. S.; Singer, K.; McDonald, I. R. Mol. Phys. 1981, 44, 135.

(101) Cipriani, P.; Nardone, M.; Ricci, F. P.; Ricci, M. A. Mol. Phys. 2001, 99, 301.

(102) Merker, T.; Engin, C.; Vrabec, J.; Hasse, H. J. Chem. Phys. 2010, 132, 234512.

(103) Harris, J. G.; Yung, K. H. J. Phys. Chem. 1995, 99, 12021.

(104) Brodholt, J.; Wood, B. Am. Mineral. 1993, 78, 558.

(105) Panhuis, M. I. H.; Patterson, C. H.; Lynden-Bell, R. M. Mol. Phys. 1998, 94, 963.

(106) Potoff, J. J.; Siepmann, J. I. AIChE J. 2001, 47, 1676.

(107) Zhang, Z. G.; Duan, Z. H. J. Chem. Phys. 2005, 122, 214507.

(108) Nguyen, T. X. Ph.D. thesis, The Univeristy of Queensland, Brisbane, 2006.

(109) Merker, T.; Vrabec, J.; Hasse, H. J. Chem. Phys. 2008, $129,087101$.

(110) Zhang, Z.; Duan, Z. J. Chem. Phys. 2008, 129, 087102.

(111) Potoff, J. J.; Errington, J. R.; Panagiotopoulos, A. Z. Mol. Phys. 1999, 97, 1073.

(112) Kolafa, J.; Nezbeda, I.; Lisal, M. Mol. Phys. 2001, 99, 1751.

(113) Lisal, M.; William, W. R. S.; Nezbeda, I. Fluid Phase Equilib. 2001, 181, 127.

(114) Vorholz, J.; Harismiadis, V. I.; Rumpf, B.; Panagiotopoulos, A. Z.; Maurer, G. Fluid Phase Equilib. 2000, 170, 203.

(115) Cui, S. T.; Cochran, H. D.; Cummings, P. T. J. Phys. Chem. B 1999, 103, 4485.

(116) Destrigneville, C. M.; Brodholt, J. P.; Wood, B. J. Chem. Geol. 1996, 133, 53

(117) Somasundaram, T.; Panhuis, M. I. H.; Lynden-Bell, R. M.; Patterson, C. H. J. Chem. Phys. 1999, 111, 2190.

(118) Song, W.; Biswas, R.; Maroncelli, M. J. Phys. Chem. A 2000, 104, 6924.

(119) Samios, S.; Stubos, A. K.; Papadopoulos, G. K.; Kanellopoulos, N. K.; Rigas, F. J. Colloid Interface Sci. 2000, 224, 272.

(120) Koh, C. A.; Montanari, T.; Nooney, R. I.; Tahir, S. F.; Westacott, R. E. Langmuir 1999, 15, 6043.

(121) Salaniwal, S.; Cui, S. T.; Cummings, P. T.; Cochran, H. D. Langmuir 1999, 15, 5188.

(122) Salaniwal, S.; Cui, S. T.; Cochran, H. D.; Cummings, P. T. Ind. Eng. Chem. Res. 2000, 39, 4543.

(123) Salaniwal, S.; Cui, S. T.; Cochran, H. D.; Cummings, P. T. Langmuir 2001, 17, 1773.

(124) Salaniwal, S.; Cui, S. T.; Cochran, H. D.; Cummings, P. T. Langmuir 2001, 17, 1784. 
(125) Anderson, K. E.; Siepmann, J. I. J. Phys. Chem. B 2008, 112, 11374 .

(126) Persson, R. A. X. J. Chem. Phys. 2011, 134, 034312.

(127) Fedchenia, I. I.; Schroder, J. J. Chem. Phys. 1997, 106, 7749.

(128) Domanski, K. B.; Kitao, O.; Nakanishi, K. Mol. Simul. 1994, $12,343$.

(129) Shen, J. W.; Kitao, O.; Nakanishi, K. Fluid Phase Equilib. 1996, 120, 81 .

(130) Steinebrunner, G.; Dyson, A. J.; Kirchner, B.; Huber, H. J. Chem. Phys. 1998, 109, 3153.

(131) Bukowski, R.; Sadlej, J.; Jeziorski, B.; Jankowski, P.; Szalewicz, K. J. Chem. Phys. 1999, 110, 3785.

(132) Bock, S.; Bich, E.; Vogel, E. Chem. Phys. 2000, 257, 147.

(133) Tsuzuki, S.; Uchimaru, T.; Mikami, M.; Tanabe, K.; Sako, T.; Kuwajima, S. Chem. Phys. Lett. 1996, 255, 347.

(134) Tsuzuki, S.; Tanabe, K. Comput. Mater. Sci. 1999, 14, 220.

(135) Allen, M. P.; Tildesley, D. J. Computer Simulation of Liquids; Oxford University Press: Oxford, U.K., 1987.

(136) Frenkel, D.; Smit, B. Understanding Molecular Simulation, 2nd ed.; Academic Press: London, 2002.

(137) De Leeuw, S. W.; Perram, J. W.; Smith, E. R. Proc. R. Soc. A 1980, 373, 27.

(138) Onsager, L. J. Am. Chem. Soc. 1936, 58, 1486.

(139) Wolf, D.; Keblinski, P.; Phillpot, S. R.; Eggebrecht, J. J. Chem. Phys. 1999, 110, 8254.

(140) Avendaño, C.; Gil-Villegas, A. Mol. Phys. 2006, 104, 1475.

(141) Gray, C. G.; Gubbins, K. E. Theory of Molecular Fluids; Clarendon Press: Oxford, U.K., 1984; Vol. 1.

(142) Span, R.; Wagner, W. J. Phys. Chem. Ref. Data 1996, 25, 1509.

(143) Thermophysical Properties of Fluid Systems; NIST Chemistry WebBook; NIST Standard Reference Database, Number 69; NIST: Gaithersburg, MD; http://webbook.nist.gov/chemistry/fluid/.

(144) Zhou, S. Z.; Solana, J. R. Phys. Chem. Chem. Phys. 2009, 11,11528

(145) Press, W. H.; Teukolsky, S. A.; Vetterling, W. T.; Flannery, B. P. Numerical Recipes in Fortran, 2nd ed.; Cambridge Univeristy Press: Cambridge, U.K., 1992.

(146) Forte, E.; Llovell, F.; Vega, L. F.; Trusler, J. P. M.; Galindo, A. J. Chem. Phys. 2011, 134, 154102.

(147) Torrie, G. M.; Valleau, J. P. Chem. Phys. Lett. 1974, $28,578-581$.

(148) Berg, B. A.; Neuhaus, T. Phys. Rev. Lett. 1992, 68, 9.

(149) Landau, D. P.; Binder, K. A Guide to Monte Carlo Simulations in Statistical Physics, 2nd ed.; Cambridge University Press: Cambridge, UK, 2005.

(150) Fitzgerald, M.; Picard, R. R.; Silver, R. N. Europhys. Lett. 1999, 46, 282.

(151) Fitzgerald, M.; Picard, R. R.; Silver, R. N. J. Stat. Phys. 2000, $98,321$.

(152) Wang, J. S.; Swendsen, R. H. J. Stat. Phys. 2002, 106, 245.

(153) Errington, J. R. J. Chem. Phys. 2003, 118, 9915.

(154) Errington, J. R. Phys. Rev. E 2003, 67, 012102.

(155) Ferrenberg, A. M.; Swendsen, R. H. Phys. Rev. Lett. 1988, 61, 2635.

(156) Ferrenberg, A. M.; Swendsen, R. H. Phys. Rev. Lett. 1989, 63, 1195.

(157) Binder, K. Phys. Rev. A 1982, 25, 1699.

(158) Potoff, J. J.; Panagiotopoulos, A. Z. J. Chem. Phys. 2000, 112,6411 .

(159) Gloor, G. J.; Jackson, G.; Blas, F. J.; de Miguel, E. J. Chem. Phys. 2005, 123, 134703.

(160) Lagache, M.; Ungerer, P.; Boutin, A.; Fuchs, A. H. Phys. Chem. Chem. Phys. 2001, 3, 4333.

(161) Lagache, M. H.; Ungerer, P.; Boutin, A. Fluid Phase Equilib. 2004, 220, 211.

(162) Callen, H. B. Thermodynamics and An Introduction to Thermostatistics; Wiley: New York, 1985.

(163) Wegner, F. J. Phys. Rev. B 1972, 5, 4529.
(164) Vega, L.; de Miguel, E.; Rull, L. F.; Jackson, G.; McLure, I. A. J. Chem. Phys. 1992, 96, 2296.

(165) Singh, J. K.; Kofke, D. A.; Errington, J. R. J. Chem. Phys. 2003, $119,3405-3412$.

(166) Bruce, A. D.; Wilding, N. B. Phys. Rev. Lett. 1992, 68, 193.

(167) Wilding, N. B. Phys. Rev. E 1995, 52, 602.

(168) $\mathrm{AAD} \%=1 / N_{\mathrm{p}} \Sigma_{i}^{N_{\mathrm{p}}}\left|\left(X_{i, \exp }-X_{i, \text { theo }}\right) / X_{i, \exp }\right| \times 100$.

(169) Guggenheim, E. A. J. Chem. Phys. 1945, 13, 253.

(170) Colina, C. M.; Lisal, M.; Siperstein, F. R.; Gubbins, K. E. Fluid Phase Equilib. 2002, 202, 253.

(171) Barker, J. A.; Henderson, D. Rev. Mod. Phys. 1976, 48, 587.

(172) Carnahan, N.; Starling, K. E. J. Chem. Phys. 1969, 51, 635.

(173) Boublik, T. Mol. Phys. 1986, 59, 775.

(174) Smith, W. Mol. Simul. 2006, 32, 933. 